\title{
Lectures on Topological Quantum Field Theory
}

\author{
DANIEL S. FREED \\ Department of Mathematics \\ University of Texas at Austin
}

December 9, 1992

What follows are lecture notes about Topological Quantum Field Theory. While the lectures were aimed at physicists, the content is highly mathematical in its style and motivation. The subject of Topological Quantum Field Theory is young and developing rapidly in many directions. These lectures are not at all representative of this activity, but rather reflect particular interests of the author.

Our theme is locality and gluing laws. The idea that global quantities can be computed from local formulas is an old one in topology and geometry. So too are formulas which express the change in a global invariant when we change a space locally. One should think of the Mayer-Vietoris sequence in homology theory $[\mathrm{Sp}]$ as an example. So too the local index theorem $[\mathrm{ABP}]$. In knot theory one has the skein relations $[\mathrm{K}]$. The Casson invariant obeys a surgery formula, and some recent work exploits formulas which calculate the change of Donaldson's invariant under certain surgeries [GM]. These examples illustrate that the gluing laws are not simply a theoretical nicety, but rather a useful tool for computation.

The idea of these lectures, then, is to explore the origin of locality in field theory from first principles. Since this notion relies on Feynman's path integral in the quantum theory, we restrict ourselves to a very simple toy model in which the path integral is a finite sum. Thus we avoid all of the analytical difficulties usually encountered in quantum theory. The penalty we pay is that this toy model has little real mathematical or physical interest. The payoff is that it nicely illustrates some of the main ideas, and allows us to explore some more subtle features. There is also locality and gluing laws in classical field theory, and in Lecture \#4 we discuss a particularly illuminating case.

Here is a brief summary of the lectures. In Lecture \#1 we give a rough description of the definition and properties of a lagrangian field theory. We state them as "axioms", though they should not be taken as a rigid mathematical system like Euclidean geometry. The detailed axioms are slightly

The author is supported by NSF grant DMS-8805684, an Alfred P. Sloan Research Fellowship, a Presidential Young Investigators award, and by the O'Donnell Foundation. He also received generous hospitality from the Geometry Center at the University of Minnesota while these notes were completed.

These are notes from 5 lectures given at the GIFT Summer School on Recent Problems in Mathematical Physics which was held in Salamanca, Spain, June, 1992. Quisiera expresar mi agradecimiento a Alberto Ibort y Miguel Rodriguez por haberme invitado a dar estas charlas y por su magnifica hospitalidad en Salamanca. 
different in different theories. Our intent is only to coerce the reader into formulating familiar theories in terms which will help him or her relate to the more mathematical theories which follow. Lectures \#2 and \#3 discuss gauge theory with finite gauge group, our toy model. Lecture \#4 is an introduction to the rich geometry of classical Chern-Simons theory. Lecture \#5 is a report on some computer computations designed to convince skeptics that it all works!

A more complete treatment of the topics in Lectures \#2-\#5 may be found in several papers of the author listed in the bibliography. That fact alone should dispel any illusion of impartiality or completeness of these lectures! 


\section{Lecture \#1: The Basic Structure of Field Theories}

Today we begin with a general discussion of basic features of field theories, both on the classical and quantum levels. We only focus on the very basic features which interest us in our discussion of a special type of field theories: topological field theories. Missing is any discussion of local analytic properties, specifics about the Lorentz metric, etc. Rather, following Segal $[\mathrm{S}]$ and Atiyah $[\mathrm{A}]$, we list a general set of axioms which field theories are meant to satisfy. They resemble the Steenrod axioms for homology theory [Sp] much more closely than the Wightman axioms for field theory [GJ]. By no means are they meant to capture all properties of field theory, just as one could hardly do intricate calculations in homology theory just knowing the axioms. But they do provide a focus for our discussion, and they emphasize the properties of interest. We present them in the context of general field theories, and illustrate them with some non-topological examples. We encourage the reader to think about these axioms in familiar examples of field theories (and also mechanical systems, which are the $0+1$ dimensional case of a field theory). There is a much more detailed and precise treatment of these axioms in [Q1].

\section{Fields and the Action}

We speak of a $(d+1)$-dimensional field theory: $d$ space dimensions and 1 time dimension. So $d=0,1, \ldots$ is the dimension of space - it is fixed at the beginning. I will always use ' $X$ ' to denote a spacetime - some sort of $d+1$ dimensional manifold - and ' $Y$ ' to denote a space - some sort of $d$ dimensional manifold. By "sort" of manifold we mean manifold with extra structure. This can take the form of topological data, like an orientation or spin structure, or differential geometric data, like a metric or conformal structure. Usual field theories are often done in $X=\mathbb{R}^{d+1}$ and $Y=\mathbb{R}^{d}$, or perhaps in some open subsets of these Euclidean spaces. A key feature of topological theories is that $X$ and $Y$ are allowed to be curved manifolds. The spacetimes $X$ are more fundamental than the spaces $Y$, so I'll talk now only about spacetimes. In traditional field theory one sometimes considers $X=S^{d+1}$, a $(d+1)$-dimensional sphere, or $X=S^{1} \times \cdots \times S^{1}$, a $(d+1)$-dimensional torus. These are then thought of as compactifications of $\mathbb{R}^{d+1}$. But we want to consider more complicated spaces.

We generally restrict to compact spacetimes, both with and without boundary.

Definition. A manifold is called closed if it is compact and has no boundary.

This is a different use of 'closed' from the 'open' and 'closed' sets of topology. Most field theories are defined on manifolds endowed with a metric, traditionally of Lorentz signature. The topological theory we consider in Lectures \#2-\#5 (Chern-Simons Theory) does not use a metric - in fact, the spacetimes have no continuous parameters. However, our first example today, the $\sigma$-model, is of the traditional type; it is defined using a metric. We use Riemannian metrics, so do Euclidean field theory.

\section{$\sigma$-model}

Usually a field theory begins with some fixed data, independent of any spacetimes, fields, etc. 
Here this is:

$$
\begin{aligned}
d=0,1, \ldots & \text { dimension of the theory minus one } \\
(M, h) & \text { a Riemannian manifold with metric } h
\end{aligned}
$$

These data are fixed once and for all. $M$ is not to be confused with the spacetimes, which are

$$
\left(X^{d+1}, g\right) \text { a compact, oriented Riemannian manifold of dimension } d+1 \text { with metric } g \text {. }
$$

Now to have a field theory we need fields! These are some sort of function on the spacetime, though the function may be vector-valued or even more twisted. For example, in gauge theory the field is a connection on the spacetime, though it is not immediately obvious how to think of this as a function on spacetime. In the $\sigma$-model the fields are:

$$
\mathcal{C}_{X}=\operatorname{Map}(X, M)
$$

These are the fields on the spacetime $X$. It is useful to think about the assignment

$$
X \longmapsto \mathcal{C}_{X}
$$

which attaches a space of fields to every spacetime. In this case a field is a smooth map $\phi: X \rightarrow M$. The other key ingredient is the action, which is a function on the space of fields:

$$
S_{X}: \mathcal{C}_{X} \rightarrow \mathbb{R}
$$

In the typical case this function has real values, but in later lectures when we consider the ChernSimons theory this will have to be modified. The $\sigma$-model action is:

$$
S_{X}(\phi)=\int_{X}|d \phi|^{2} d \operatorname{vol}_{X}
$$

It might look more familiar if we introduce local coordinates $x^{i}$ on $X$ and $y^{\alpha}$ on $M$. Then $\phi$ looks like $y^{\alpha}=y^{\alpha}\left(x^{1}, \ldots, x^{d+1}\right)$ and

$$
S_{X}(\phi)=\int_{X} \frac{\partial y^{\alpha}}{\partial x^{i}} \frac{\partial y^{\beta}}{\partial x^{j}} h_{\alpha \beta} g^{i j} \sqrt{\operatorname{det}\left(g_{i j}\right)} d x^{1} \ldots d x^{d+1} .
$$

We require $X$ compact to avoid convergence problems.

\section{Formal Axioms}

What I want to observe here are formal properties of the fields and action, properties which carry over to any field theory. We will not discuss the $\sigma$-model any further; we only introduced the $\sigma$-model action here to illustrate the general structure of field theories. So these axioms apply to any field theory whose spacetimes are compact oriented Riemannian manifolds. They need only slight modifications, depending on the particular types of spacetimes in the theory, to apply to any field theory. 
Functoriality Axiom. Suppose $f:\left(X^{\prime}, g^{\prime}\right) \rightarrow(X, g)$ is an orientation preserving isometry. Thus $f: X^{\prime} \rightarrow X$ is a diffeomorphism of manifolds which preserves the orientation, and $f^{*}(g)=g^{\prime}$. We allow the possibility $X=X^{\prime}$ and $g=g^{\prime}$; then $f$ is an isometry of $(X, g)$. Think of these as symmetries of spacetimes. Then $f$ induces a map of fields

$$
f^{*}: \mathcal{C}_{X} \rightarrow \mathcal{C}_{X^{\prime}}
$$

and the action is preserved:

$$
S_{X^{\prime}}\left(f^{*} \phi\right)=S_{X}(\phi), \quad \phi \in \mathcal{C}_{X}
$$

This is the axiom which we need to modify depending on the type of theory. Generally, the map $f$ should be a diffeomorphism which preserves whatever extra geometric and topological structures the spacetimes carry.

Exercise 1 Check this for the $\sigma$-model action (2). In local coordinates it is the change of variable formula for the integral.

Exercise 2 If $X=\mathbb{R}^{4}$ with the Minkowski metric, what are the isometries $f: X \rightarrow X$ ? What does the Functoriality Axiom tell us about the action on Minkowski space?

Exercise 3 A compact 1-manifold which is connected is diffeomorphic to either a circle or a closed interval. Now determine the possible metrics on the manifold up to isometry. (Hint: Construct invariants of the metric which are unchanged by isometry.)

Exercise 4 Suppose $d=0$. Then the spacetime $X$ is an interval or a circle, and a field is a path in $M$. Interpret the action (2). Is the length of the path a possible action?

Orientation Axiom. If $-X$ denotes $X$ with the opposite orientation, then

$$
\mathcal{C}_{-X}=\mathcal{C}_{X}
$$

and

$$
S_{-X}(\phi)=-S_{X}(\phi), \quad \phi \in \mathcal{C}_{X}
$$

This is ultimately an expression of the unitarity of the theory, and may not hold in nonunitary theories. In the simple theory we discuss in Lecture \#2 the spacetimes do not even carry an orientation.

The next axiom explains how to express the fields and the action for nonconnected spacetimes in terms of the fields and action for connected spacetimes. 
Additivity Axiom. Suppose $X_{1}, X_{2}$ are spacetimes and $X=X_{1} \sqcup X_{2}$ the disjoint union. Then

$$
\mathcal{C}_{X} \cong \mathcal{C}_{X_{1}} \times \mathcal{C}_{X_{2}}
$$

Let $\phi_{1} \in \mathcal{C}_{X_{1}}, \phi_{2} \in \mathcal{C}_{X_{2}}$ and $\phi=\phi_{1} \sqcup \phi_{2} \in \mathcal{C}_{X}$. Then

$$
S_{X}(\phi)=S_{X_{1}}\left(\phi_{1}\right)+S_{X_{2}}\left(\phi_{2}\right) .
$$

Exercise 5 Verify (3) and (4) for the $\sigma$-model.

The final axiom is a generalization of the Additivity Axiom, and is the central idea of these lectures. I call it the Gluing Axiom - it expresses the idea that the action and the fields are local.

Gluing Axiom. Let $X$ be a spacetime and $Y \hookrightarrow X$ an oriented codimension one submanifold. Cut along $Y$ to obtain a new spacetime $X^{\text {cut }}$ (see Figure 1 ). Then there is an "equalizer diagram"

$$
\mathcal{C}_{X} \rightarrow \mathcal{C}_{X^{\text {cut }}} \rightrightarrows \mathcal{C}_{Y}
$$

If $\phi \in \mathcal{C}_{X}$ with $\phi^{\text {cut }} \in \mathcal{C}_{X^{\text {cut }}}$ the corresponding field on $X^{\text {cut }}$, then

$$
S_{X}(\phi)=S_{X^{\text {cut }}}\left(\phi^{\text {cut }}\right)
$$

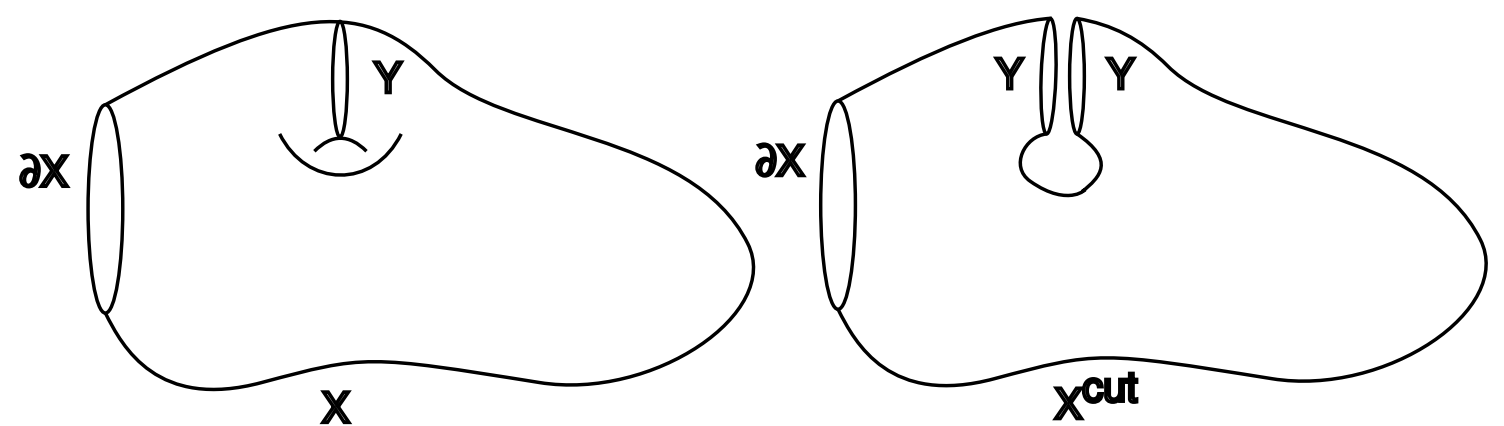

Figure 1: Cutting a manifold along a codimension one submanifold

What does (5) mean? It means that a field on $X$ is a field on $X^{\text {cut }}$ which has the same boundary values on the two components of $\partial X^{\text {cut }}$ corresponding to $Y$. (Incidentally, this is very similar to the defining property of a sheaf.) What (6) means is that the action on $X$ can just as well be compared on $X^{\text {cut }}$. To simplify notation we will often picture cutting and pasting as in Figure 2, where we cut a closed manifold into two pieces. Then what the preceding means in that figure is that if we 

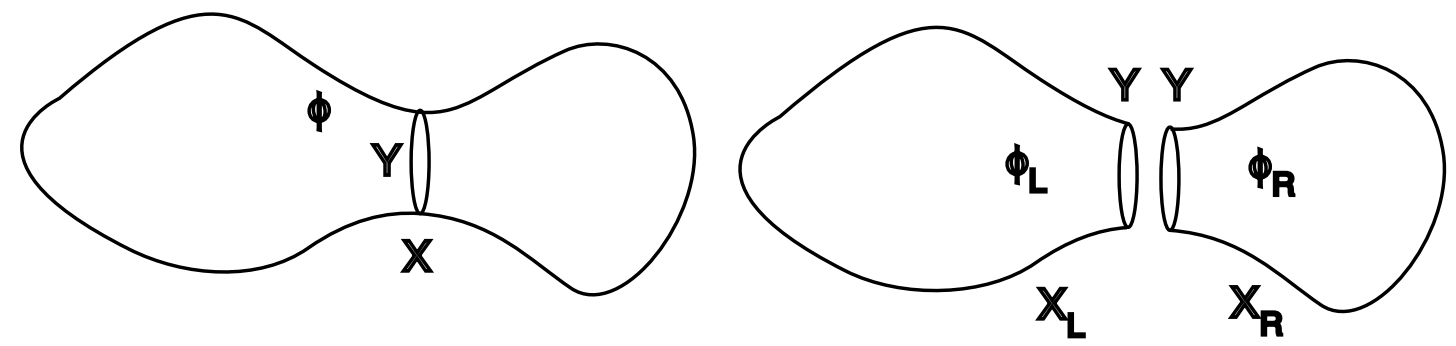

Figure 2: Cutting a closed manifold into two pieces

have fields $\phi_{L} \in \mathcal{C}_{X_{L}}$ and $\phi_{R} \in \mathcal{C}_{X_{R}}$ which agree on $Y$, then there is a field $\phi \in \mathcal{C}_{X}$ which agrees with these on each piece, and the action is

$$
S_{X}(\phi)=S_{X_{L}}\left(\phi_{L}\right)+S_{X_{R}}\left(\phi_{R}\right) .
$$

We will see these pictures many times throughout these lectures.

Exercise 6 Derive (7) from (6). You will need to use the Additivity Axiom as well.

Exercise 7 Verify the Gluing Law for the $\sigma$-model action. Check the special case of $d=0$.

The four axioms are characteristic properties of the classical action of a field theory. We do not mean the above to be a rigorous statement of the axioms - we will have to modify them anyway in the Chern-Simons Theory. Nor do we wish to develop the subject axiomatically. Rather, we wish to isolate and emphasize these properties.

\section{Topological Actions}

A topological field theory is defined on spacetimes with no continuous parameters, i.e., no differential geometric data like a (background) metric. The spacetimes may carry discrete topological data like an orientation or spin structure. Of course, the fields may still have continuous parameters.

We give a typical example of the form of a topological action. This is a simple variant of the topological actions which arise in practice. The fixed data for this theory is:

$$
\begin{aligned}
d=0,1, \ldots & \text { dimension of the theory minus one } \\
M & \text { a smooth manifold } \\
\omega \in \Omega_{M}^{d+1} & \text { a }(d+1) \text {-form on } M
\end{aligned}
$$

Again, $M$ is not to be confused with the spacetimes, which we now list together with the fields and the action.

Spacetimes: $X$ a compact oriented $(d+1)$-manifold.

Fields: $\mathcal{C}_{X}=\operatorname{Map}(X, M)$ 
Action: If $\phi: X \rightarrow M$ then the action is

$$
S_{X}(\phi)=\int_{X} \phi^{*} \omega
$$

So this theory is a sort of topological version of the $\sigma$-model in $M$, though it is not what is usually referred to as the Topological Sigma Model [W2].

Exercise 8 Verify the axioms stated above for this action. What is the proper statement of the Functoriality Axiom?

Exercise 9

a) Suppose that $\omega$ is closed, i.e., $d \omega=0$. Show that if $\partial X=0$ ( $X$ is closed $)$, then $S_{X}(\phi)$ only depends on the homotopy class of $\phi$.

b) If $X$ is not closed, then $S_{X}(\phi)$ varies as $\phi$ moves through a homotopy. Nonetheless, we can reinterpret the action as an invariant of the homotopy class. Try to do this by explaining the dependence of the action on $\phi$. (Hint: The action only depends on the boundary values of $\phi$.)

The Chern-Simons action may be considered as a variant of (8), but the symbols ' $\omega$ ' and ' $\int$ ' have to be radically reinterpreted.

\section{Quantum Theory}

The path integral quantization requires a measure $\mu_{X}$ on $C_{X}$ which satisfies certain properties which we do not specify here. Then for $X$ closed we define the partition function as the path integral

$$
Z_{X}=\int_{\mathcal{C}_{X}} e^{i S_{X}(\phi)} d \mu_{X}(\phi)
$$

Note that $Z_{X}$ is a complex number.

The big mathematical problem is that in many examples of interest $\mu_{X}$ has not been rigorously defined. This has been a big subject of investigation. Also, in Euclidean field theory the ' $i$ ' is replaced by ' -1 ' and we integrate $e^{-S_{X}(\phi)}$ instead of $e^{i S_{X}(\phi)}$. You can add a parameter $\hbar$ if you like. Here my concern is only the formal properties, which for now do not include the properties as $\hbar \rightarrow 0$. We return to that perturbation theory in Lecture \#5 for the Chern-Simons theory. Here we only consider formal properties of the exact path integral.

If $\partial X \neq \phi$ then $Z_{X}$ is a function of a field on the boundary $\partial X$. So if $\psi \in \mathcal{C}_{\partial X}$ let

$$
\mathcal{C}_{X}(\psi)=\left\{\phi \in \mathcal{C}_{X}: \partial \phi=\psi\right\}
$$

That is, $\mathcal{C}_{X}(\psi)$ consists of fields on $X$ whose boundary value is $\psi$. Then

$$
Z_{X}(\psi)=\int_{\mathcal{C}_{X}(\psi)} e^{i S_{X}(\phi)} d \mu_{X}(\phi)
$$


The function $Z_{X}$ belongs to a vector space $E(\partial X)$ of "certain" functions on $\mathcal{C}_{\partial X}$ - this is the quantum Hilbert space. The construction of $E(\partial X)$ is the subject of canonical quantization. We discuss it in the Chern-Simons theory in Lecture \#4, where we will explain a little about what 'certain' means above.

As an example, consider the (Euclidean) $\sigma$-model for $d=0$. The classical action in this theory is the energy of a path on a Riemannian manifold $M$. The quantum field theory is the usual quantum mechanics on $M$. A "space" in this theory, which might occur as (part of) the boundary of a spacetime, is simply a point. The quantum Hilbert space is

$$
E(p t)=\mathcal{F}(M)
$$

the space of functions on $M$. Think of smooth functions; here I won't bother with technicalities about exactly what functions on $M$ we ought to consider. A spacetime which is connected is either a closed interval or a circle, endowed with a metric. For the interval $X_{t}=[0, t]$ we obtain $Z_{X_{t}}(x, y)=p_{t}(x, y)$ depending on points $x, y \in M$, which comprise the fixed field on the boundary $\partial([0, t])=\{t\} \cup-\{0\}$. (The minus sign is for the orientation.) This $p_{t}$ for the Euclidean theory is the heat kernel of $M$, the probability density which measures the amount of heat which flows from $x$ to $y$ in time $t$. If $H: \mathcal{F}(M) \rightarrow \mathcal{F}(M)$ is the Laplace operator we can write this heat operator as

$$
Z_{[0, t]}=e^{-t H}: \mathcal{F}(M) \rightarrow \mathcal{F}(M)
$$

The fundamental property of gluing is illustrated by:

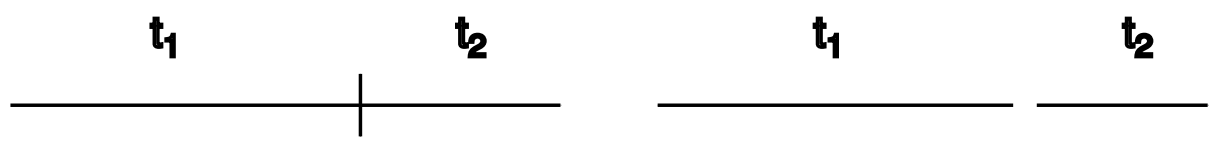

Figure 3: $e^{-\left(t_{1}+t_{2}\right) H}=e^{-t_{1} H} e^{-t_{2} H}$

Another case of gluing is the following:

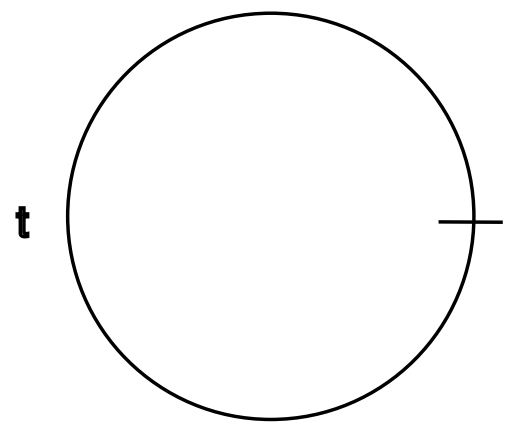

Figure 4: $Z_{S^{1}(t)}=\operatorname{Tr} e^{-t H}$

That is, the partition function for the circle is the trace of the heat kernel, depending on the length $t$ of the circle. 


\section{Axioms for Quantum Theory}

The properties of the quantum Hilbert spaces $E(Y)$ associated to any space $Y$ ( $d$-manifold + extra structure) and the generalized partition functions (path integrals) $Z_{X}$ associated to a spacetime $X$ $((d+1)$-manifold + extra structure $)$ mirror those mentioned above for the classical action. Indeed, they can be made to formally follow from them if one assumes the existence of a measure on the space of fields with appropriate properties. These axioms were stated by Segal [S] for conformal field theory and Atiyah [A] for topological theories. But they are quite general - the only difference between different types of theories is the structure of the manifold and so the symmetries in the Functoriality Axiom.

¡Repetimos! A field theory is formulated on spacetimes $X^{d+1}$ and spaces $Y^{d}$ with certain extra structure (orientation, metric, ...) depending on the type of theory. There may also be some fixed data. Our spacetimes and spaces are compact. The basic objects in the quantum theory are the quantum Hilbert space and the path integral.

Quantum Hilbert Space:

Path Integral:

$$
\begin{gathered}
Y \longmapsto E(Y) \quad(\partial Y=\phi) \\
X \longmapsto Z_{X} \in E(\partial X)
\end{gathered}
$$

The path integral over a closed manifold is also called the partition function. The term 'correlation function' is often used when the boundary is nontrivial, though we tend to (mis) use the term 'partition function' instead. The Hilbert spaces and path integrals satisfy properties analogous to those of the classical theory.

Functoriality Axiom. If $f: Y^{\prime} \rightarrow Y$ is a diffeomorphism preserving the extra structure (orientation, metric, ...) then there is an induced map

$$
f_{*}: E\left(Y^{\prime}\right) \rightarrow E(Y)
$$

If $f: X^{\prime} \rightarrow X$ is a diffeomorphism preserving the extra structure with induced boundary map $\partial f: \partial X^{\prime} \rightarrow \partial X$ then

$$
(\partial f)_{*}\left(Z_{X^{\prime}}\right)=Z_{X}
$$

This says that symmetries of the spaces are implemented on the Hilbert spaces, and that symmetries of the spacetimes preserve the path integrals. In many quantum field theories, including the ChernSimons theory, this is not correct as it stands - the symmetries only act projectively. This means that the composition of symmetries acts as the composition of the individual actions up to an overall phase. In other terms, it means that a central extension of the symmetries acts on the Hilbert spaces. Often this is used to disqualify a quantum field theory as unrealistic. The study of these central extensions and projective factors goes under the name anomalies, and has been the subject of much mathematical investigation and understanding. 


\section{Orientation Axiom.}

$$
\begin{aligned}
E(-Y) & \cong \overline{E(Y)} \quad \text { (conjugate Hilbert space) } \\
Z_{-X} & =\overline{Z_{X}} .
\end{aligned}
$$

Multiplicativity Axiom.

$$
\begin{aligned}
E\left(Y_{1} \sqcup Y_{2}\right) & \cong E\left(Y_{1}\right) \otimes E\left(Y_{2}\right) \quad \text { (tensor product) } \\
Z_{X_{1} \sqcup X_{2}} & =Z_{X_{1}} \otimes Z_{X_{2}}
\end{aligned}
$$

The multiplicativity axiom expresses the behavior of the quantum Hilbert spaces and path integrals under disjoint union of spaces and spacetimes.

The final, and for us the most important axiom, is a generalization of (15) which tells how the path integral behaves when we glue manifolds along an oriented closed codimension one submanifold, as depicted in Figure 5.

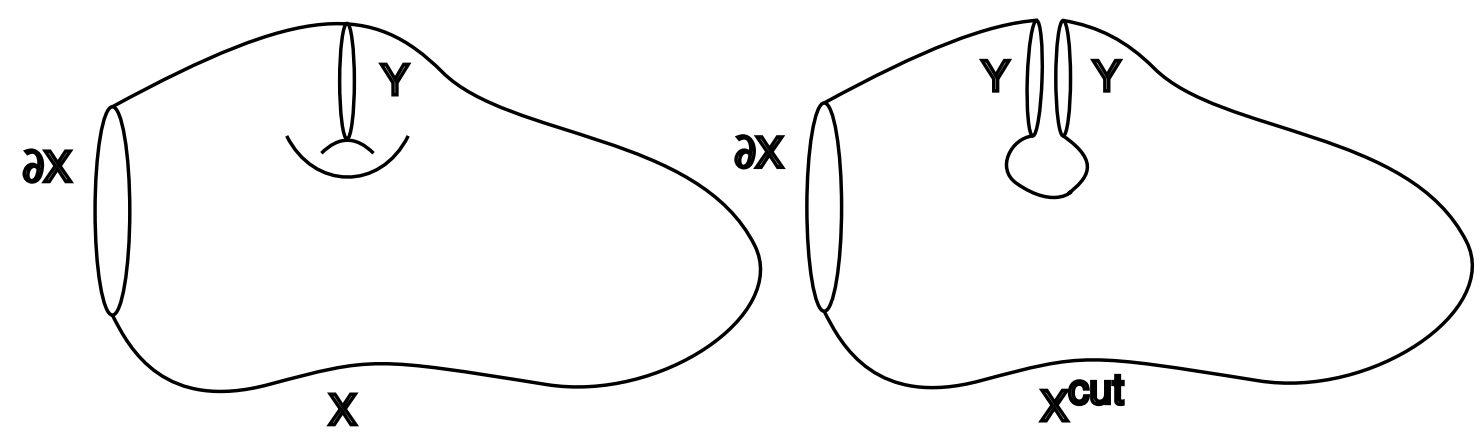

Figure 5: Cutting a manifold along a codimension one submanifold

Gluing Law. Suppose $Y \hookrightarrow X$ is a closed oriented codimension one submanifold, and $X^{\text {cut }}$ the spacetime obtained by cutting along $Y$. Then we have the path integrals $Z_{X} \in E(\partial X)$ and

$$
Z_{X^{\text {cut }}} \in E\left(\partial X^{\text {cut }}\right)=E(\partial X \sqcup Y \sqcup-Y) \cong E(\partial X) \otimes E(Y) \otimes \overline{E(-Y)} .
$$

Now there is a trace

$$
\begin{gathered}
\operatorname{Tr}_{Y}: E\left(\partial X^{\text {cut }}\right) \quad \cong E(\partial X) \otimes E(Y) \otimes \overline{E(Y)} \longrightarrow E(\partial X) . \\
\backslash \text { contract using inner product }
\end{gathered}
$$

The axiom asserts that the path integrals satisfy

$$
Z_{X}=\operatorname{Tr}_{Y} Z_{X^{\mathrm{cut}}}
$$


Exercise 10 Show that quantum mechanics (11) and (12) satisfies these axioms.

Exercise 11 Think of other (relativistic) field theories in higher dimensions and try to see what properties of these the axioms capture.

\section{Exercise 12}

a) What does the gluing axiom say about $Z_{[0, t]}$ in a topological $0+1$ dimensional theory?

b) More generally, compute $Z_{[0,1] \times Y^{d}}$ in a $d+1$ dimensional topological theory.

c) Also, compute $Z_{S^{1} \times Y^{d}}$ in a $d+1$ dimensional topological theory. 


\section{Lecture \#2: Gauge Theory with a Finite Gauge Group}

Let $G$ be a finite group. For example, $G$ could be the cyclic group $\mathbb{Z} / n \mathbb{Z}$ of $n$ elements, or the symmetric group $S_{n}$ of $n$ ! elements. There is no restriction on $G$. We construct, in arbitrary dimensions, a gauge theory with structure group $G$. It is surely the simplest quantum field theory there is, and is an excellent toy model to illustrate certain features. It exhibits the algebraic and geometric structure we are interested in, but has no analytic difficulties whatsoever: all integrals in the theory are finite sums. It also illustrates some basic properties of gauge theory, especially the role of symmetries and reducible connections.

This lecture is based on joint work with Frank Quinn [FQ]. The theory discussed here first appeared in a paper of Dijkgraaf and Witten [DW]. There is related unpublished work by Segal, Kontsevich, and Bernstein/Kazhdan. More recent work [F5] examines finer algebraic structures in this theory, in particular the connection to quantum groups, which is a topic of many other lectures at this Summer School.

\section{Fields}

Let $X$ be a spacetime. For this theory we only need a "bare" $(d+1)$-manifold, assumed compact. No orientation, metric, etc. is needed. The space of fields is

$$
\mathcal{C}_{X}=\left\{\begin{array}{l}
P \\
\downarrow \\
X
\end{array}: P \text { is a principal (Galois, regular) covering space with structure group } G .\right\}
$$

In other terms, $P$ is a principal bundle with structure group $G$. Thus $G$ acts freely on $P$ and the quotient is $P / G=X$. We always take $G$ to act on the right.

As an example, consider $G=\mathbb{Z} / 3 \mathbb{Z}$. If $X=p t$ then picture is

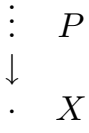

with $G$ cyclically permuting the 3 points. If $X=S^{1}$ then there are 3 possibilities, up to isomorphism, as illustrated in Figure 6. The nontrivial coverings are pictured as pieces of helices, but the endpoints are meant to be identified. Topologically, the total space $P$ in these covers is a circle. 

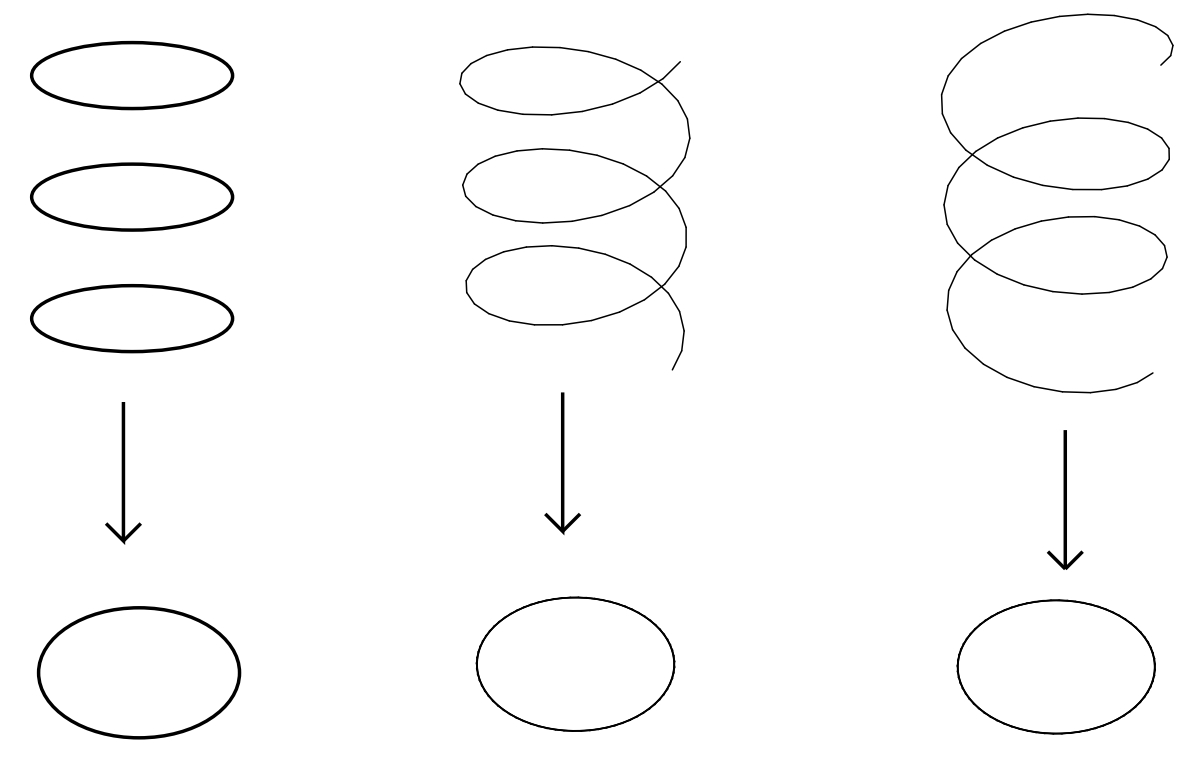

Figure 6: Triple Coverings of $S^{1}$

As opposed to the $\sigma$-model, where we have a nice space of fields, the fields in (17) do not technically form a topological space or even a set. (The collection of all of anything is not a set.) But there is a notion of isomorphic fields, and the fields in our theory fall into a finite number of isomorphism classes.

Definition. An isomorphism $\varphi$ from $P^{\prime}$ to $P$ is a map $\varphi: P^{\prime} \rightarrow P$ which commutes with the $G$ action and such that the induced map on the quotient $X$ is the identity.

This means that for $p^{\prime} \in P^{\prime}$ and $g \in G$ we have $\varphi\left(p^{\prime} \cdot g\right)=\varphi\left(p^{\prime}\right) \cdot g$, where the first '.' indicates the $G$ action on $P^{\prime}$ and the second '.' the $G$ action on $P$. Any such map induces a map $\bar{\varphi}: X \rightarrow X$, and we restrict our isomorphism to have $\bar{\varphi}=$ id. More generally, we can consider diagrams

$$
\begin{array}{ccc}
P^{\prime} & \stackrel{\varphi}{\rightarrow} & P \\
\downarrow & & \downarrow \\
X^{\prime} & \stackrel{\varphi}{\rightarrow} & X
\end{array}
$$

They enter when we consider symmetries of the spacetimes as well as symmetries of the fields.

So we get a picture of $\mathcal{C}_{X}$ which we schematically render in Figure 7. Each point is a field and each arrow is a symmetry of fields, that is, an isomorphism. We can compose two arrows if the second one starts where the first one ends. Notice that every object has an identity automorphism. (An automorphism is an isomorphism which starts and ends at the same place). Also, every arrow has an inverse. The arrows which start and end at an object $P$ form a group called Aut $P$, the automorphism group of $P$. There is a finite number of arrows from any point to any other. Aut $P$ is also called the group of gauge transformations of $P$. 

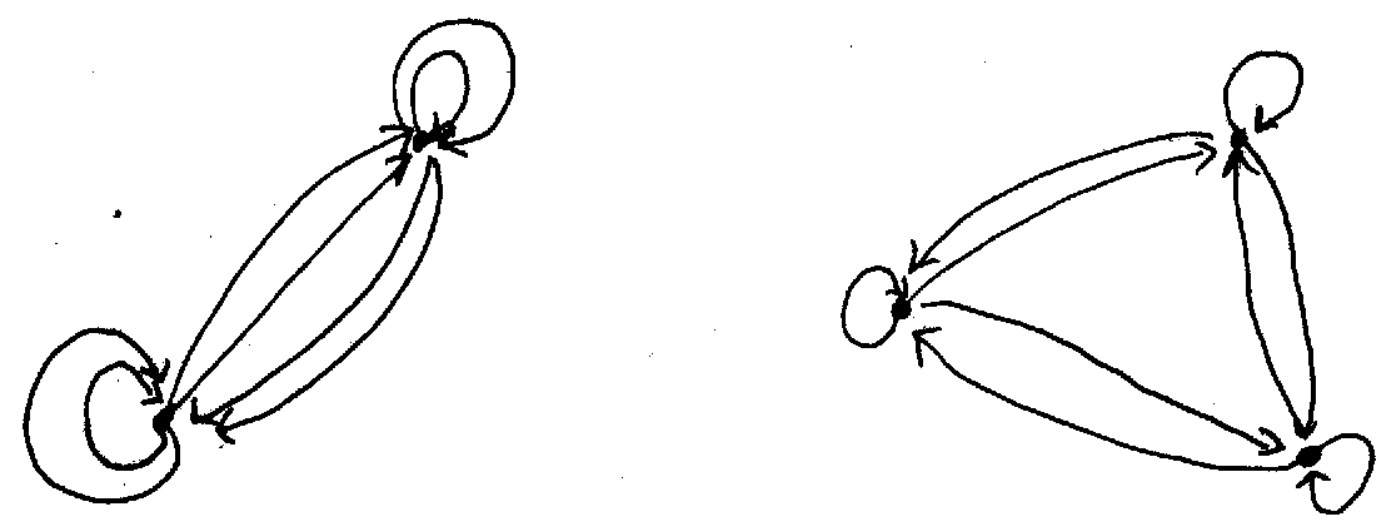

Figure 7: Configuration space for gauge theory with finite gauge group

We remark that this picture applies to the space of (gauge) fields in any gauge theory, except that there are now continuous parameters. The technical word for this structure is a groupoid. See $[\mathrm{F} 1, \S 1]$ for a discussion.

The fields $P$ and $P^{\prime}$ are equivalent $\left(P \cong P^{\prime}\right)$ or isomorphic if there is an arrow between them. Let

$$
\overline{\mathcal{C}_{X}}=\text { set of equivalence classes of fields on } X \text {. }
$$

What makes gauge theories with finite gauge group so simple is that $\overline{\mathcal{C}_{X}}$ is a finite set if $X$ is compact.

Exercise 13 Determine $\overline{\mathcal{C}_{X}}$ for $X=p t$. For $X=S^{1}$.

Exercise 14 Show that $\mathcal{C}_{X}$ satisfies the formal axioms listed in Lecture \#1. Does $\overline{\mathcal{C}_{X}}$ satisfy these axioms? (Notice that there is no orientation axiom in this theory.)

Exercise 15 Show that $\overline{\mathcal{C}_{X}}$ is a finite set for any compact manifold $X$.

Suppose $X$ is connected. Fix a basepoint $x \in X$ and $p \in P_{x}$, where $P_{x}$ is the fiber of $P$ at $x$. Then a field $P \rightarrow X$ determines a map

$$
\text { loops at } x \rightarrow G
$$

by taking the holonomy around the loop using the basepoint $p$. (See Figure 8.) Any loop at $x$ lifts uniquely to a path in $P$ which starts at $p$ and ends at some $p^{\prime}$ in the fiber $P_{x}$ of $P$ over $x$. The 


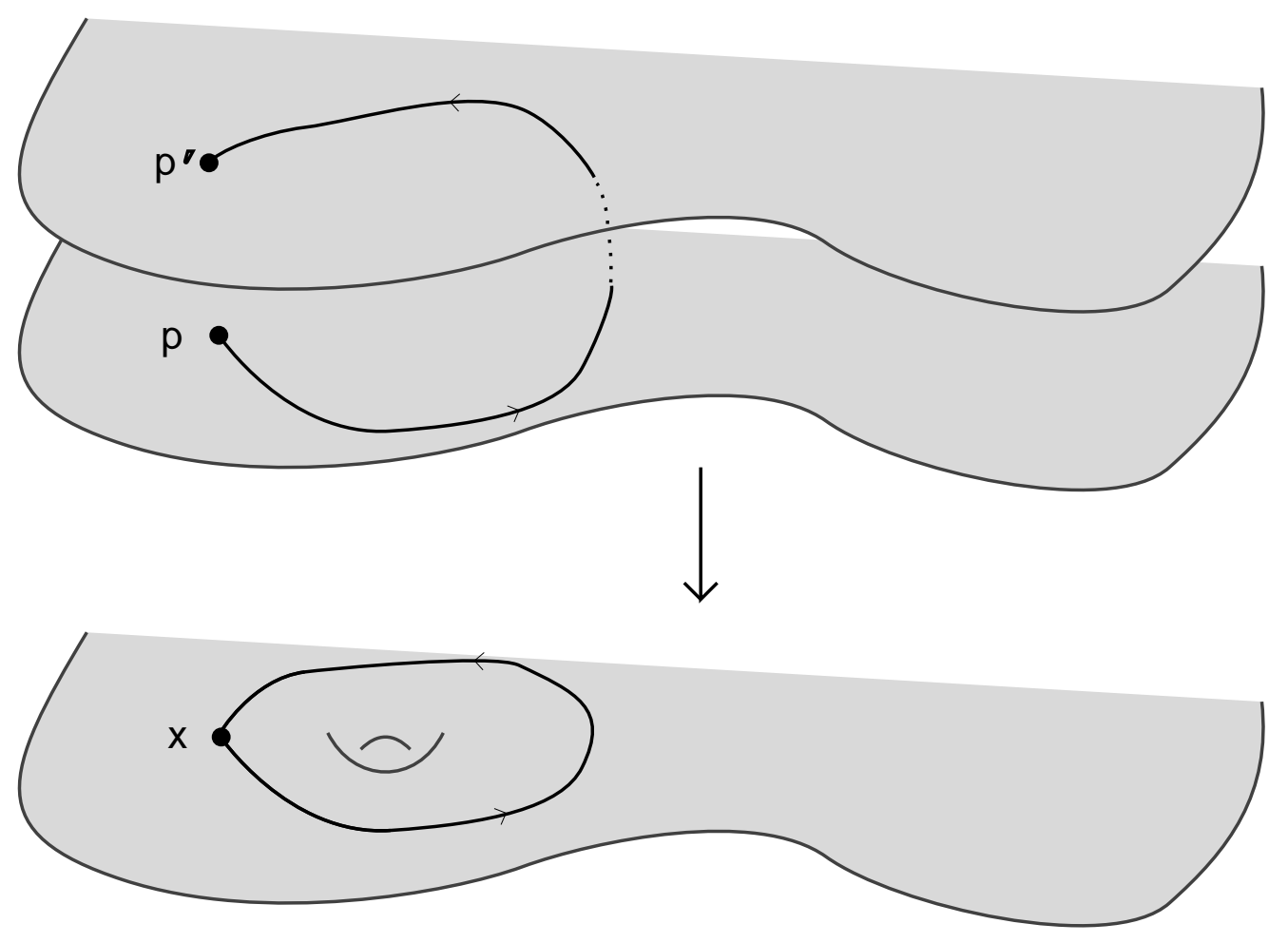

Figure 8: Definition of holonomy

holonomy $h \in G$ is the unique $h$ satisfying $p^{\prime}=p \cdot h$. The holonomy only depends on the homotopy class of the loop.

Exercise 16

a) Check this last assertion.

b) Show that the map $\pi_{1}(X, x) \rightarrow G$ defined by holonomy is a homomorphism of groups.

c) If $\gamma$ is this homomorphism, and we change the basepoint $p$ and/or $x$, then the new homomorphism is $g \gamma g^{-1}$ for $g \in G$.

Exercise 16 holds more generally for any flat connection with arbitrary gauge group.

The above exercise shows that there is an isomorphism (of sets)

$$
\overline{\mathcal{C}_{X}} \cong \operatorname{Hom}\left(\pi_{1}(x), G\right) / G
$$

if $X$ is connected. (We omit the irrelevant basepoint.)

In Exercise 14 it is important to observe that the fields $\mathcal{C}_{X}$ are local, i.e., they can be cut and glued. 


\section{Action}

After so much work just to define fields, we can relax — we define the action to be identically zero!

$$
S_{X}(P)=0 \text { for all } P \in \mathcal{C}_{X}
$$

There are other possibilities, but this requires using the cohomology of the classifying space of the group, or equivalently group cohomology. These "twisted theories" are in essence constructed through a modification of (8) and the exercises which follow. See [FQ], [Q1], [Q2] for details.

So the classical action in our toy theory is completely trivial! It's surprising that we can learn anything from such an action. The fact that we will demonstrates the rich structure already present in the symmetries of the fields.

Now on to the quantization.

\section{Measure}

Since $\mathcal{C}_{X}$ is discrete, a measure is just an assignment of a mass to each field $P$. The appropriate choice is

$$
\mu_{X}(P)=\frac{1}{\# \operatorname{Aut} P}
$$

The '\#' sign counts the number of elements in a set. Notice that if $P \cong P^{\prime}$ then Aut $P \cong$ Aut $P^{\prime}$. So $\mu$ passes to a measure on the quotient $\overline{\mathcal{C}_{X}}$.

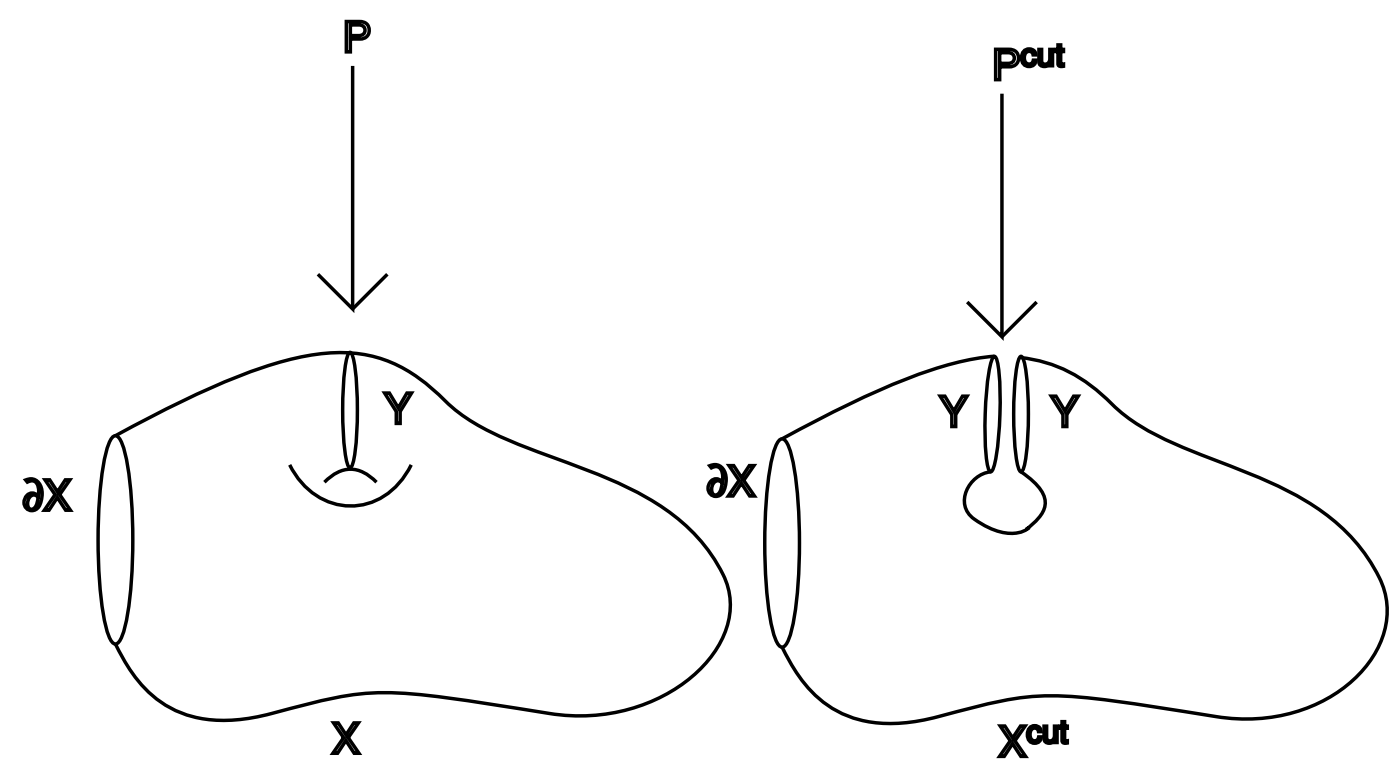

Figure 9: Gluing bundles 
We did not state axioms for the measure in Lecture \#1. But we do need to know how the measure behaves under gluing. Fix a bundle $Q \rightarrow Y$. We consider the gluing map

$$
g_{Q}: \overline{\mathcal{C}_{X^{\text {cut }}}}(Q \sqcup Q) \rightarrow \overline{\mathcal{C}_{X}(Q)}
$$

from (equivalence classes of) bundles over $X^{\text {cut }}$ whose restriction to each copy of $Y$ is $Q$ to (equivalence classes of) bundles over $X$. (This needs to be made precise in a careful way.) The key formula is that for any $[P] \in \overline{\mathcal{C}_{X}}$ whose restriction to $Y$ is isomorphic to $Q$,

$$
\mu_{X}([P])=\operatorname{vol}\left(g_{Q}^{-1}([P])\right) \cdot \mu_{X}(Q)
$$

We use $[P]$ to denote the equivalent classes of bundles containing $P$.

As an illustration consider gluing the two ends of an interval together to obtain a circle. Then $Q$ is the trivial bundle

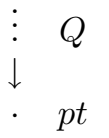

Now the equivalence classes of bundles over an interval are in $1: 1$ correspondence with the elements of $G$, since we are meant to have isomorphisms of the restriction to each endpoint with the trivial bundle, so can identify the holonomy, as in Figure 10. Finally, the equivalence classes of bundles over the circle are in 1: 1 correspondence with the conjugacy classes in $G$. This happens since when we glue the two ends of the interval together we are supposed to forget the basepoint and the trivialization, so we can only determine the holonomy up to conjugacy. Therefore, for this particular gluing (20) specializes to the map

$$
g: G \rightarrow \operatorname{Conj}(G)
$$

which assigns to each $h \in G$ its conjugacy class $[h]$. Then (21) reduces to a standard formula in group theory:

$$
\#[h] \cdot \# C_{h}=\# G \text {. }
$$

Here $C_{h}$ is the centralizer of $h$, i.e., the subgroup of elements of $G$ which commute with $h$, and $h$ is an arbitrary element in the conjugacy class $[h]$.

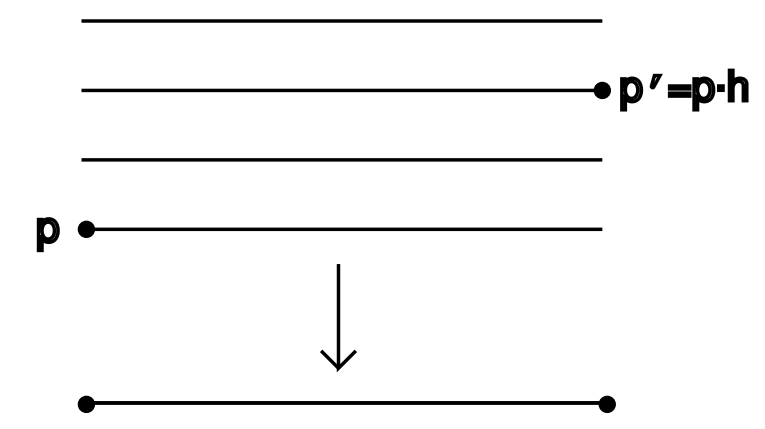

Figure 10: Bundle with basepoints over the interval 


\section{Quantization}

Given the fields, the action, and the measure we can carry out the quantization using (9) and (10). Keep in mind that the set of equivalence classes of bundles is finite, so we are dealing with finite measure spaces. In other words, the "path integral" in this case is a finite sum. Also, note that since there are symmetries on the fields, we carry out the path integral on the equivalence classes of fields. Note too that the space of equivalence classes is not the quotient of a set by a group action. Rather, the picture looks like Figure 7.

That said, the quantum Hilbert space is

$$
E(Y)=L^{2}\left(\overline{\mathcal{C}_{Y}}, \mu_{Y}\right)
$$

and the path integral on a closed manifold is

$$
Z_{X}=\int_{\overline{\mathcal{C}_{X}}} e^{i S_{X}([P])} d \mu_{X}([P])=\operatorname{vol}\left(\overline{\mathcal{C}_{X}}\right)
$$

If $X$ has boundary, then for each $Q \in \mathcal{C}_{\partial X}$ we let $\overline{\mathcal{C}_{X}}(Q)$ be the set of bundles over $X$ whose restriction to $\partial X$ is $Q$, up to isomorphisms which are the identity on $\partial X$. If $X$ has no closed components, then elements of $\overline{\mathcal{C}_{X}}(Q)$ have no automorphisms. Define

$$
Z_{X}(Q)=\int_{\overline{\mathcal{C}_{X}}(Q)} e^{i S_{X}([P])} d \mu_{X}([P])=\operatorname{vol}\left(\overline{\mathcal{C}_{X}}(Q)\right)
$$

This is the standard procedure: we integrate over fields which satisfy a boundary condition. It is easy to check that for equivalent fields $Q \cong Q^{\prime}$ on $Y$, we have $Z_{X}(Q)=Z_{X}\left(Q^{\prime}\right)$. So $Z_{X}$ is a function on the set of equivalence classes of fields, that is, it is an element of $E(\partial X)$.

Theorem. Equations (22)-(24) define a topological quantum field theory.

That is, these definitions satisfy the axioms stated in Lecture \#1.
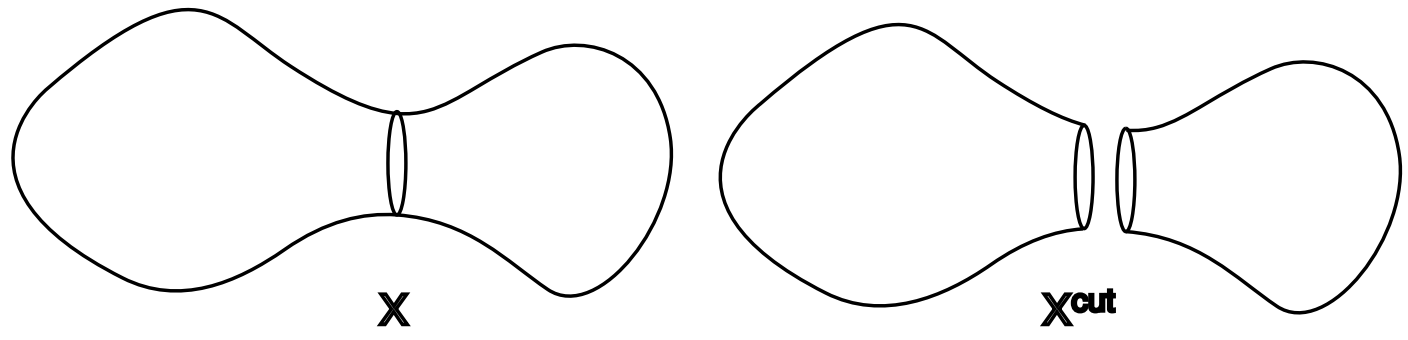

Figure 11: A simple gluing 
We don't write a complete proof, but do illustrate the gluing law in case $\partial X=\emptyset$ (Figure 11). (As usual, this simplification is only for ease of notation.) Let $\{Q\}$ be a set of representations of $\overline{\mathcal{C}_{Y}}$. Recall the gluing map (20). Then

$$
\begin{aligned}
Z_{X} & =\operatorname{vol}\left(\overline{\mathcal{C}_{X}}\right) \\
& =\sum_{Q \in\{Q\}} \int_{\overline{\mathcal{C}_{X}}(Q)} d \mu_{X}([P]) \\
& \left.=\sum_{Q \in\{Q\}}\left\{\int_{\overline{\mathcal{C}_{X} \text { cut }}(Q \sqcup Q)} d \mu_{X^{\text {cut }}}\left(\left[P^{\text {cut }}\right]\right)\right\} \cdot \mu_{Y}(Q) \quad \text { by }(21)\right) \\
& =\sum_{Q \in\{Q\}} Z_{X^{\text {cut }}}(Q \sqcup Q) \mu_{Y}(Q) \\
& =\operatorname{Tr}_{Y} Z_{X^{\text {cut }}} .
\end{aligned}
$$

Exercise 17 Carefully verify the theorem above.

\section{Consequence of the Axioms}

We note a few elementary properties of topological quantum field theories in general. First, let $I$ be a closed interval (it doesn't matter which - they are all diffeomorphic) and $Y$ a space $(d$ manifold). Consider the product spacetime $I \times Y$. It is a spacetime which is globally a product of time and space. (We write time first for reasons of orientation.) Its boundary is $-Y \sqcup Y$. So using the orientation and multiplicativity axioms (13) and (14) we identify $Z_{I \times Y}$ as a map

$$
Z_{I \times Y}: E(Y) \rightarrow E(Y)
$$

Now the gluing law applied to

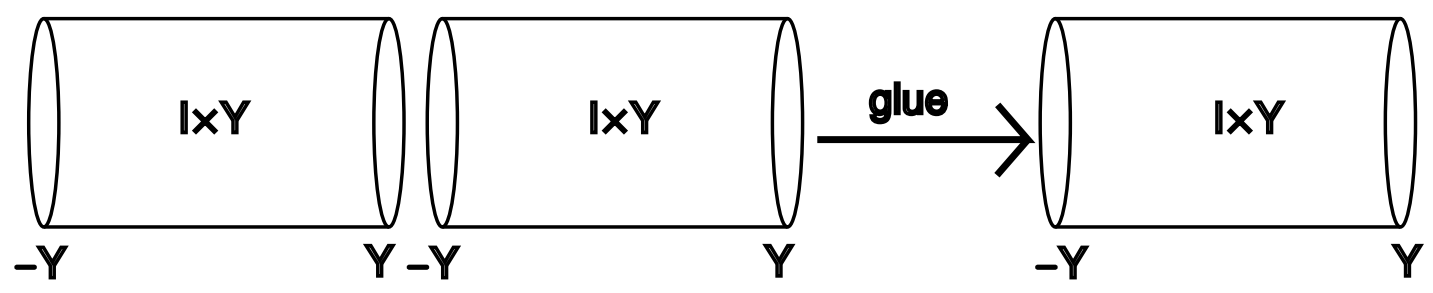

Figure 12: Gluing cylinders

implies

$$
\left(Z_{I \times Y}\right)^{2}=Z_{I \times Y}
$$

We may as well assume a nondegeneracy condition:

$$
Z_{I \times Y}=\mathrm{id}
$$


In general, (25) only implies that $Z_{I \times Y}$ is a projection operator on $E(Y)$. But only the states in the image ever appear in the theory, so it is hardly a loss of generality to deal with theories which are nondegenerate in the sense that (26) holds.

Similarly, the gluing law applied to

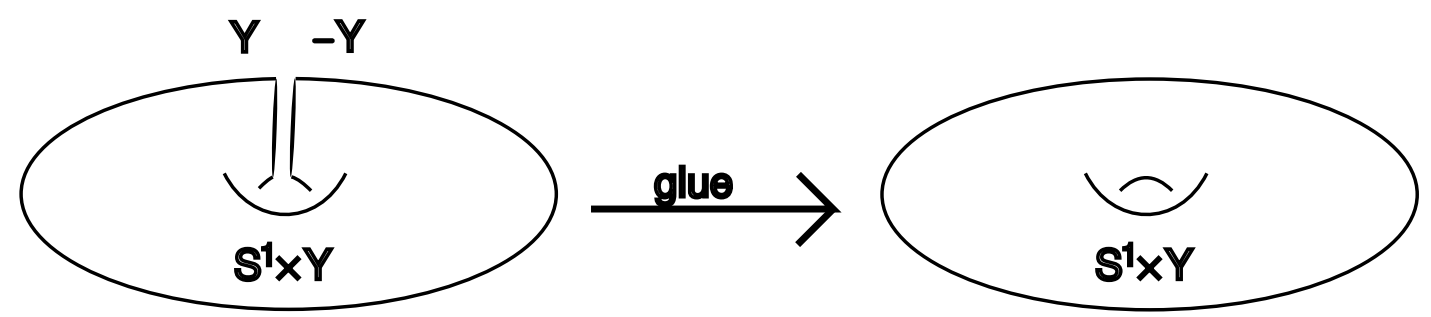

Figure 13: Gluing the ends of a cylinder

shows that

$$
Z_{S^{1} \times Y}=\operatorname{dim} E(Y)
$$

in a nondegenerate theory.

Exercise 18

a) One of the axioms states that a diffeomorphism $f: Y^{\prime} \rightarrow Y$ induces a linear map $f_{*}: E\left(Y^{\prime}\right) \rightarrow$ $E(Y)$. Show that (in a nondegenerate theory) if $f_{0}$ is homotopic to $f_{1}$, then $\left(f_{0}\right)_{*}=\left(f_{1}\right)_{*}$.

b) Suppose that we have a $2+1$ dimensional theory. Show that $E\left(S^{1} \times S^{1}\right)$ carries a representation of $S L_{2}(\mathbb{Z})$, the group of $2 \times 2$ matrices with integer entries and positive determinant which are invertible and whose inverse also has integer entries. (If there are no orientations the appropriate group is $G L_{2}(\mathbb{Z})$.)

\section{$0+1$ Dimensional Theory}

There is not much to say here. The basic vector space is $E(p t)$ and if the theory is nondegenerate, then by (26)

$$
Z_{I}=\text { identity }: E(p t) \rightarrow E(p t)
$$

By $(27), Z_{S^{1}}=\operatorname{dim} E(p t)$.

In the theory constructed from a finite group $\overline{\mathcal{C}_{p t}}$ has one element, the equivalence class of the trivial bundle. So $E(p t)$ is a 1 dimensional vector space. Now $\overline{\mathcal{C}_{S^{1}}}$ is in $1: 1$ correspondence with the conjugacy classes, so (27) implies

$$
\sum_{\substack{\text { conjugacy } \\ \text { classes }[h]}} \frac{1}{\# C_{h}}=1 .
$$

In fact, this is true and is equivalent to $(22)$. 


\section{$1+1$ Dimensional Theory}

We first discuss $1+1$ dimensional topological theories in general. Our discussion is not meant to be precise, but in a given example one can usually make it so. This material is well-known.

There is a basic vector space in the theory

$$
E=E\left(S^{1}\right) .
$$

The disk $D^{2}$ gives an element in this vector space

$$
Z_{D^{2}} \in E
$$

Recall that $E$ is also assumed to be endowed with an inner product. This really is related to the orientation, but we will ignore that here. Also, for simplicity we ignore the conjugation here and treat $E$ as a real vector space. The other crucial ingredient is the path integral over the "pair of pants" pictured in Figure 14.
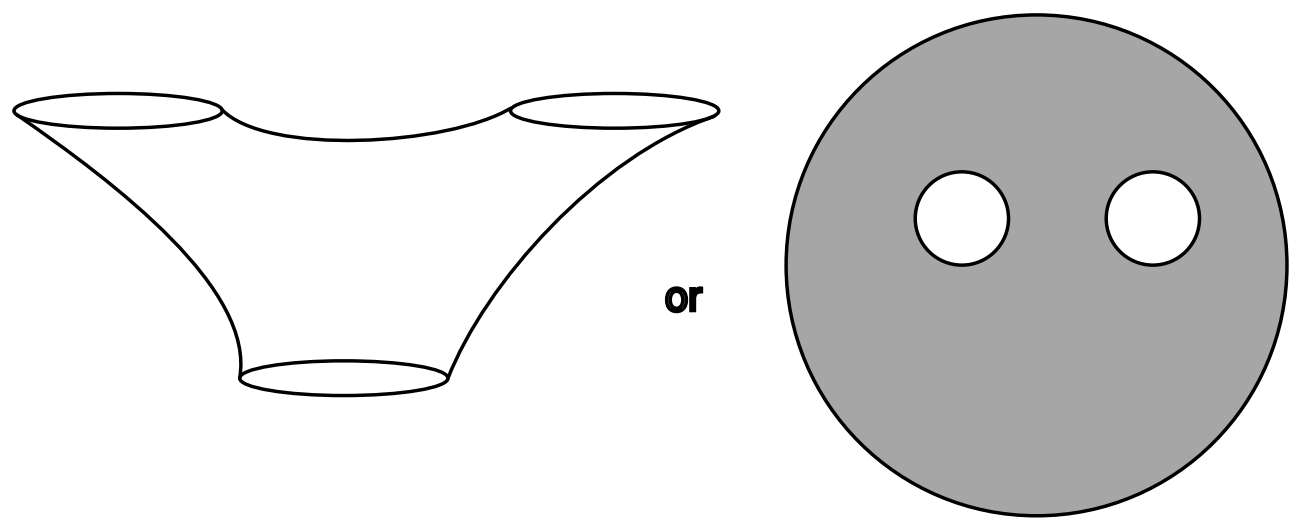

Figure 14: Pair of pants

We identify this path integral (using the inner product to identify $E \cong E^{*}$ ) as a map

$$
m: E \otimes E \rightarrow E .
$$

Proposition. The map $m$ is a multiplication which makes $E$ a commutative, associative algebra with identity element $Z_{D^{2}}$. Furthermore, $m$ is compatible with the inner product in the sense that

$$
a \otimes b \otimes c \longmapsto(m(a, b), c), \quad a, b, c \in E,
$$

is totally symmetric in $a, b, c$.

This is easy to see with pictures, and is proved using the Functoriality Axiom and the gluing law. For example, to see that $Z_{D^{2}}$ is the identity map, glue the disk to the pair of pants to obtain a cylinder: 


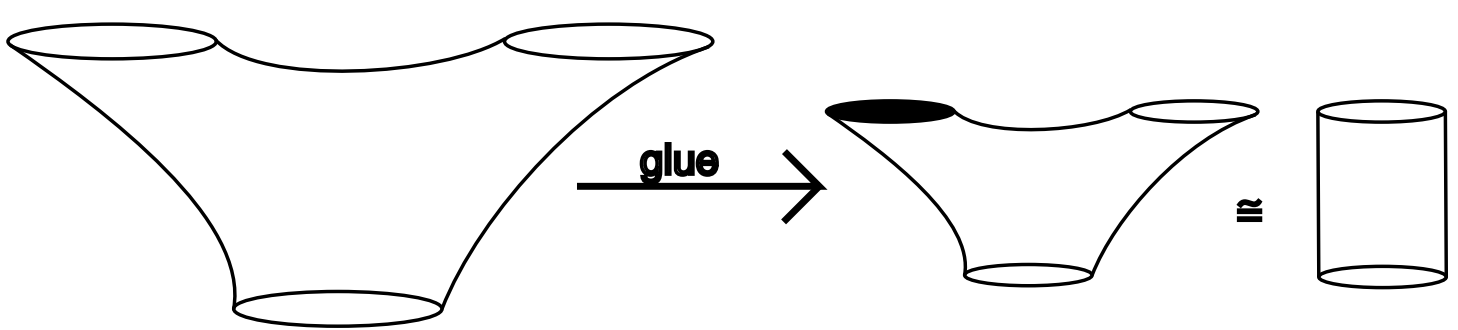

Figure 15: Multiplication by the identity element

By (26) the path integral over the cylinder is the identity map. Associativity is the equivalence of the two diagrams
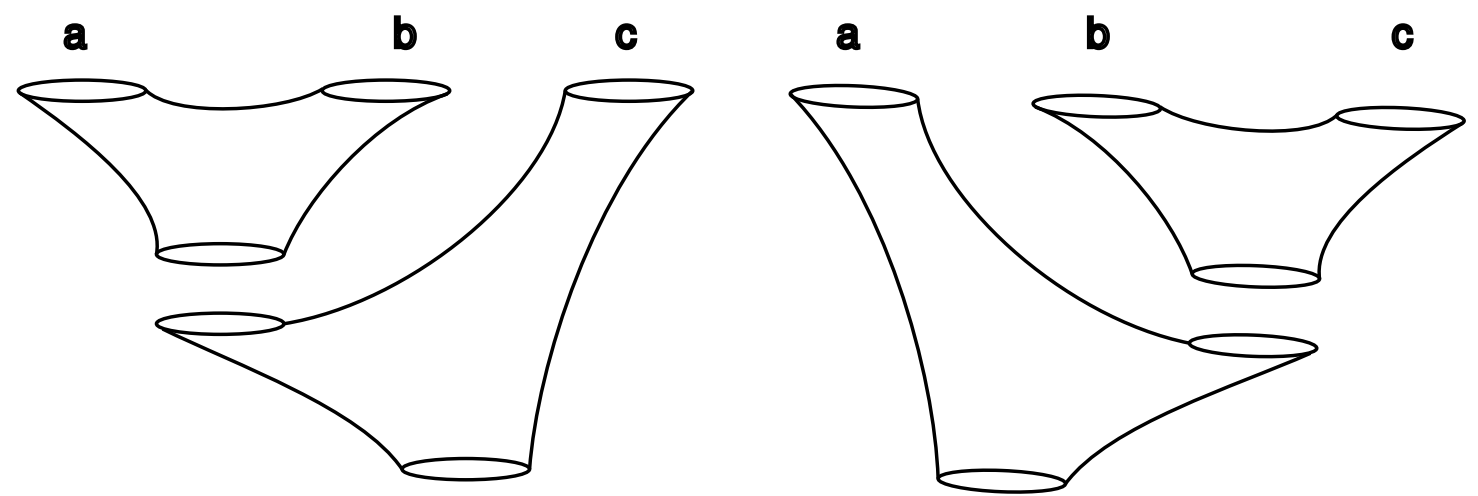

Figure 16: Associativity law

after gluing. The other assertions follow from the self-diffeomorphisms of the pair of pants (Figure 14) which permute the boundary components.

Exercise 19 Prove carefully the assertions in the proposition.

In addition, using the inner product one can show that $E$ is a semisimple algebra. This means that there is an isomorphism

$$
E \cong \mathbb{C} \times \cdots \mathbb{C}
$$

to a product of copies of $\mathbb{C}$. In other words, there exist elements $d_{i} \in E, i=1, \ldots$, dim $E$ such that

$$
d_{i} d_{j}= \begin{cases}0 & i \neq j \\ d_{j} & i=j\end{cases}
$$

These elements are unique up to order.

Exercise 20

a) Express the identity element of $E$ in terms of $d_{i}$.

b) Show that the $d_{j}$ are orthogonal. 
Up to isomorphism the algebra $E$ and inner product are determined by the numbers

$$
\lambda_{i}^{2}=\left(d_{i}, d_{i}\right)=\left|d_{i}\right|^{2} .
$$

Furthermore, the partition function of a surface $\Sigma_{g}$ of genus $g$

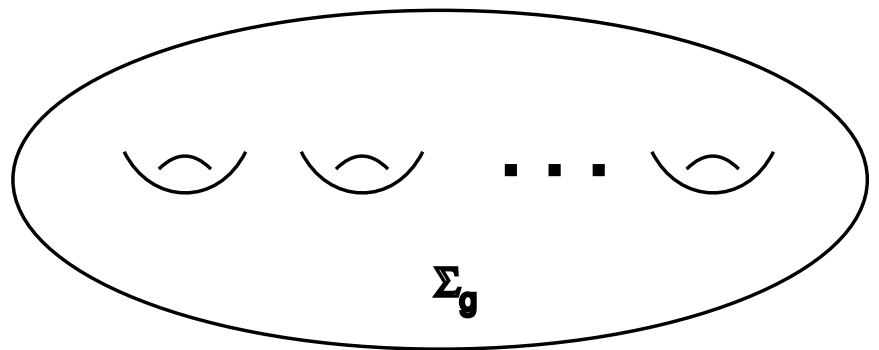

Figure 17: Surface of genus $g$

is computable in terms of the $\lambda_{i}^{2}$ as

$$
Z_{\Sigma_{g}}=\sum_{i}\left(\lambda_{i}^{2}\right)^{1-g}
$$

Exercise 21 Prove formula (29).

In the context of "conformal blocks", which we will meet in the next lecture, (29) is called the Verlinde formula. Here it is presented abstractly for $1+1$ dimensional topological field theories.

We apply this general discussion to the $1+1$ dimensional gauge theory with finite gauge group $G$. Since $\overline{\mathcal{C}_{S^{1}}}$ is in $1: 1$ correspondence with the conjugacy classes in $G$, we identify

$$
\begin{aligned}
E\left(S^{1}\right) & \cong\{\text { central functions on } G\} \\
& \cong\{\text { functions } f: G \rightarrow \mathbb{C} \text { invariant under conjugation }\}
\end{aligned}
$$

Some computation shows that the inner product is

$$
\left(f_{1}, f_{2}\right)=\frac{1}{\# G} \sum_{g \in G} f_{1}(g) \overline{f_{2}(g)} .
$$

Exercise 22 Derive (30) from the measure (19) and the definition (22).

Finally, we can do the path integral (24) over the pair of pants (Figure 14) to determine the multiplication. It turns out to be the convolution

$$
\left(f_{1} * f_{2}\right)(g)=\sum_{\substack{g_{1} g_{2}=g \\ 24}} f_{1}\left(g_{1}\right) f_{2}\left(g_{2}\right) .
$$


Exercise 23 Derive (31).

Now we can apply the Schur orthogonality relations of finite group theory to determine the special elements $d_{i}$ in (28). They are

$$
d_{i}=\frac{\operatorname{dim} V_{i}}{\# G} \cdot \chi_{i}
$$

where $V_{1}, \ldots, V_{s}$ are the irreducible representations of $G$ and $\chi_{i}$ is the character of $V_{i}$. From (29) we conclude

$$
Z_{\Sigma_{g}}=\sum_{i}\left(\frac{\operatorname{dim} V_{i}}{\# G}\right)^{2-2 g}
$$

\section{A cute formula}

As a simple application of the ideas in this lecture, and as a further illustration of the gluing law, we use (32) to derive a nice formula which counts the representations of surface groups in finite groups. Although it may hold some intrinsic interest, we present it here as a toy illustration of how the ideas in topological quantum field theory, particularly the gluing law, can be used to derive a result in a field external to quantum field theory.

To begin, recall from (23) that

$$
Z_{\Sigma_{g}}=\operatorname{vol}\left(\overline{\mathcal{C}_{\Sigma_{g}}}\right) .
$$

Let $\Gamma_{g}=\pi_{1} \Sigma_{g}$ be the surface group. Then from (18) and (19) we have

$$
Z_{\Sigma_{g}}=\sum_{[\gamma] \in \operatorname{Hom}\left(\Gamma_{g}, G\right) / G} \frac{1}{\# C_{\gamma}}
$$

where $\gamma: \Gamma_{g} \rightarrow G$ is a homomorphism, $[\gamma]$ its conjugacy class in $\operatorname{Hom}\left(\nabla_{g}, G\right) / G$, and $C_{\gamma} \subset G$ the stabilizer of $\gamma$ in $\operatorname{Hom}\left(\Gamma_{g}, G\right)$ under the conjugation action of $G$. So

$$
\begin{aligned}
Z_{\Sigma_{g}} & =\sum_{[\gamma] \in \operatorname{Hom}\left(\Gamma_{g}, G\right) / G} \frac{1}{\# C_{\gamma}} \\
& =\sum_{\gamma \in \operatorname{Hom}\left(\Gamma_{g}, G\right)} \frac{1}{\#[\gamma]} \frac{1}{\# C_{\gamma}} \\
& =\frac{1}{\# G} \# \operatorname{Hom}\left(\Gamma_{g}, G\right) .
\end{aligned}
$$

Combining (33) and (32) we find our desired "cute formula":

$$
\# \operatorname{Hom}\left(\Gamma_{g}, G\right)=(\# G)^{2 g-1} \sum_{i}\left(\operatorname{dim} V_{i}\right)^{2-2 g} .
$$

Recall that $V_{1}, \ldots, V_{s}$ is a representative list of irreducible representations of $G$. Equation (34) can be proved using standard group theoretical methods, though it is not obvious. Here we have derived it using the gluing laws of quantum field theory.

Exercise 24 Check (34) for abelian groups $G$.

Exercise 25 Check (34) for $g=0$ and $g=1$. 


\section{LECTURE \#3: $2+1$ DimensionAL THEORIES With Finite GAUGE GRoup}

The structure of $2+1$ dimensional theories has been investigated by Reshetikhin/Turaev, Kontsevich, Walker, Alvarez-Gaume, Sierra, Gomez, and many more - I am not sure of exact references. One gets the impression from these investigations that eventually there is an equivalence

$$
\left\{\begin{array}{l}
2+1 \text { dimensional TQFT with } \\
\text { stronger gluing laws than } \\
\text { stated in these lectures }
\end{array}\right\} \longleftrightarrow\left\{\begin{array}{l}
\text { "braided monoidal categories" } \\
\text { of a certain type, or some } \\
\text { sort of quantum group data }
\end{array}\right\}
$$

$$
\text { (Geometry) (Algebra) }
$$

Much attention has been devoted to constructing the geometry from the algebra. For example, the work of Reshetikhin/Turaev [RT], Walker [Wa] give precise theorems about this. Here we discuss the reverse, showing how some algebraic structures emerge out of the gluing laws of the TQFT. Rather than work in some abstract axiomatic setting, we consider the gauge theory with finite structure group $G$ that we discussed in Lecture \#2. The algebraic structures we encounter are closely related to the symmetries of the fields.

For the material in this lecture I refer to my paper [FQ] with Quinn. I also refer to Quinn's lectures [Q1], which go into more detail about the algebraic structures which arise. My recent work [F5] derives the algebraic structure (including the modular tensor categories and quantum groups) directly from the classical action.

\section{Surfaces with boundary.}

In the $2+1$ dimensional theory the "spaces" are 2 dimensional. The new point is to consider cutting and pasting (gluing) of surfaces, that is, to extend the theory to surfaces with boundary. Recall that for closed surfaces $Y$ we constructed a quantum Hilbert space $E(Y)$ in (22). Our goal is to extend this construction to compact surfaces with boundary, and then to derive a gluing law for this extension.

For a closed surface $Y$ the quantum Hilbert space $E(Y)$ consists of functions on $\overline{\mathcal{C}_{Y}}$, the set of equivalence classes of bundles over $Y$. If we want to allow surfaces with boundary, define quantum Hilbert spaces attached to these surfaces, and glue such surfaces along boundary components, then this gluing must induce a gluing on equivalence classes of bundles. On the other hand, gluing is not well-defined on equivalence classes of bundles.

Example (in lower dimensions): Up to isomorphism there is only one bundle over the interval $I$ - the trivial bundle. But if we glue the two ends of $I$ to form a circle $S^{1}$, then there are many possible bundles over $S^{1}$ obtained by gluing the trivial bundle.

To make gluing well-defined on equivalence classes we introduce basepoints. Thus a surface $Y$ with boundary is meant to have a basepoint $y$ on each component of $\partial Y$ as in Figure 18 . Then we define

$$
\mathcal{C}_{Y}^{\prime}=\left\{\left\langle Q ; q_{1}, \ldots, q_{k}\right\rangle: Q \rightarrow Y \text { is a principal } G \text { bundle and } q_{i} \in Q_{y_{i}} \cdot\right\}
$$




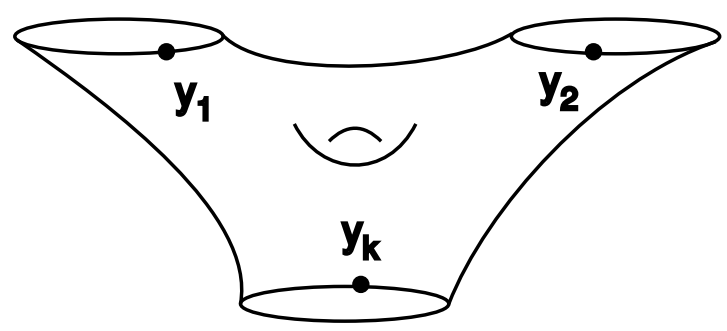

Figure 18: Surface with basepoints

Here $Q_{y_{i}}$ is the fiber of $Q$ over the basepoint $y_{i}$.

Example (in lower dimensions): A bundle over $I$ with basepoints looks like

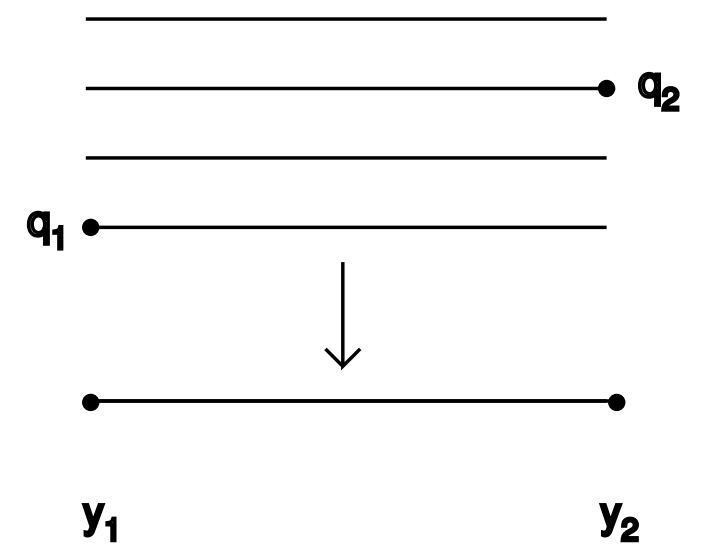

Figure 19: A principal $G$ bundle over the interval with basepoints

Now there is no ambiguity about the gluing - we identify $q_{1}$ and $q_{2}$ when we glue. If $q_{2}=q_{1} \cdot h$ for $h \in G$, then the holonomy of the glued bundle is the conjugacy class of $h$.

There is again a notion of isomorphism for elements of $\mathcal{C}_{Y}^{\prime}$-now bundle maps are required to preserve the basepoints. The set of equivalence classes (still finite) is denoted $\overline{\mathcal{C}_{Y}^{\prime}}$. As we did in the example above, we can define $\mathcal{C}_{Y}^{\prime}$ for all compact manifolds $Y$, not just for surfaces.

Exercise 26

a) Determine $\overline{\mathcal{C}_{I}^{\prime}}$ for an interval $I$.

b) Determine $\overline{\mathcal{C}_{D^{2}}^{\prime}}$ for the disk.

c) Determine $\overline{\overline{\mathcal{C}}_{I \times S^{1}}^{\prime}}$ for the cylinder.

d) Determine $\overline{\mathcal{C}_{Y}^{\prime}}$ for the pair of points $Y$.

e) Determine $\overline{\mathcal{C}_{Y}^{\prime}}$ for the surface $Y=\Sigma_{g}$ - disk. 


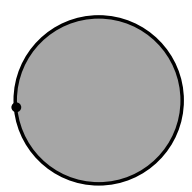

disk

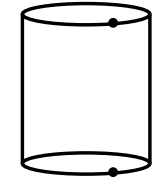

cylinder

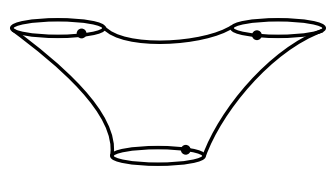

pair of pants

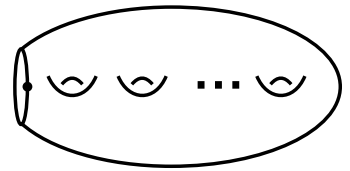

$\Sigma_{\mathrm{g}}$-disk

Figure 20: Elementary surfaces with basepoints

Exercise 27 Suppose $S$ is a connected manifold with basepoint $s \in S$. Let $R \rightarrow S$ and $R^{\prime} \rightarrow S^{\prime}$ be isomorphic $G$ bundles with basepoints $r \in R_{S}, r^{\prime} \in R_{S}^{\prime}$ in the fibers over $s$. Prove that there is a unique isomorphism $R^{\prime} \rightarrow R$ which maps $r^{\prime}$ to $r$.

This last exercise implies that elements of $\mathcal{C}_{Y}^{\prime}$ do not have nontrivial automorphisms. In other words, the picture of $\mathcal{C}_{Y}^{\prime}$ is
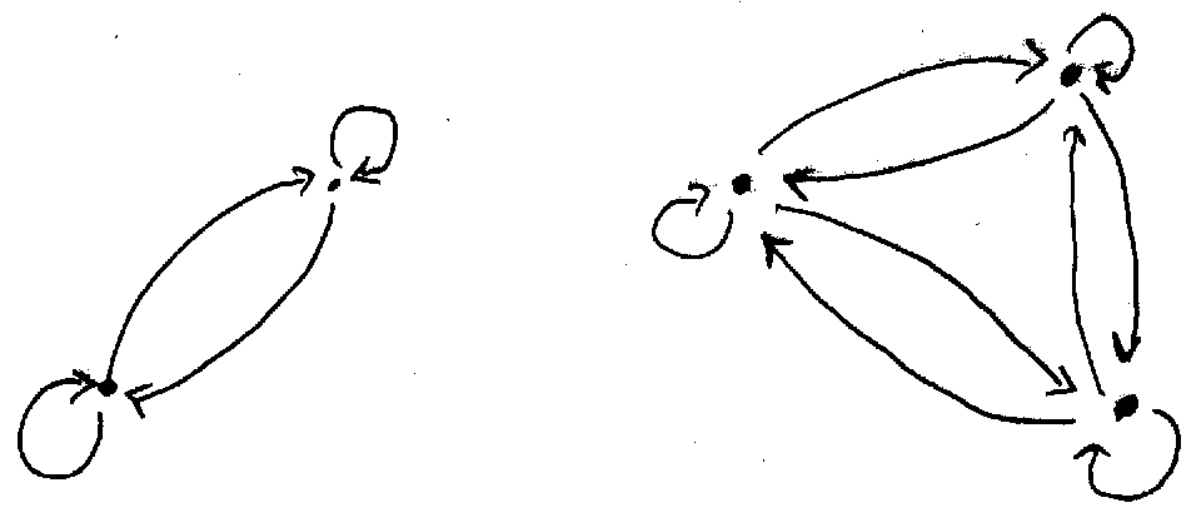

Figure 21: The configuration space for bundles with basepoints

There is a unique arrow in each direction between isomorphic bundles. This is why gluing is welldefined on the quotient.

Now a bundle over $S^{1}$ with basepoint has a definite holonomy $h \in G$. (Recall that without the basepoint the holonomy is determined only up to conjugacy.) So in Figure 22, if $Q_{1} \in \mathcal{C}_{Y_{1}}^{\prime}$ and $Q_{2} \in \mathcal{C}_{Y_{2}}^{\prime}$, then we can glue $Q_{1}$ to $Q_{2}$ along $S$ if the holonomies agree, and this gluing is well-defined on equivalence classes. Denote the glued bundle by $Q_{1} \circ Q_{2}$. Note we can glue along any components of a surface with boundary - for example, as pictured in Figure 23. As usual, we use the picture in Figure 22 only to simplify the notation. So the gluing operation is well defined as a map

$$
\begin{aligned}
& \overline{\mathcal{B}_{Y_{1}, Y_{2}}} \subset \overline{\mathcal{C}_{Y_{1}}^{\prime}} \times \overline{\overline{\mathcal{C}_{Y_{2}}^{\prime}}} \\
& g \\
& { }_{28}^{\overline{\mathcal{C}_{Y}^{\prime}}}
\end{aligned}
$$




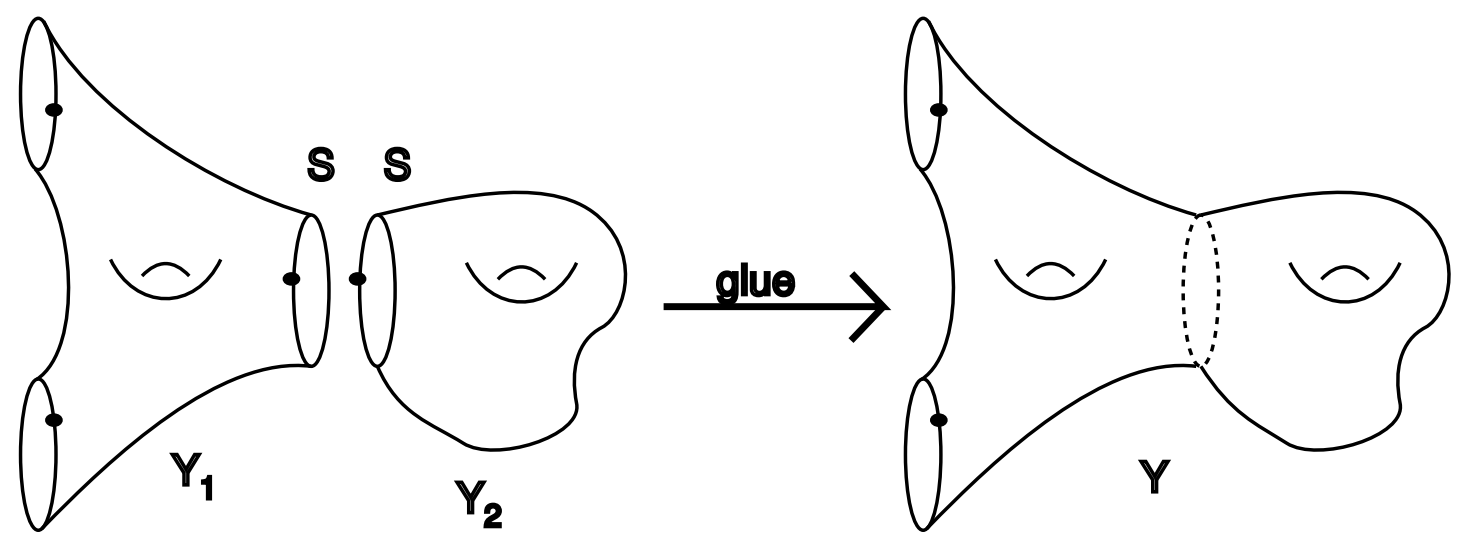

Figure 22: Gluing surfaces and bundles with basepoints

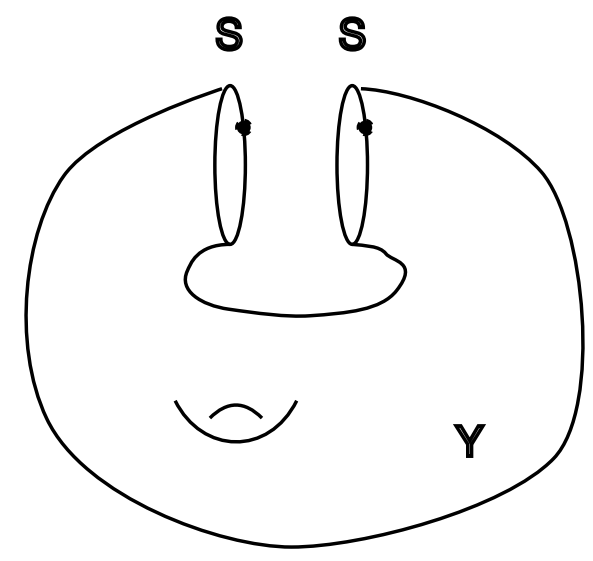

Figure 23: Another gluing of pointed surfaces

where $\mathcal{B}_{Y_{1}, Y_{2}}$ is the set of pairs $\left\langle Q_{1}, Q_{2}\right\rangle$ (with basepoints) whose holonomies agree along $S$, and $\overline{\mathcal{B}_{Y_{1}, Y_{2}}}$ is the set of equivalence classes of such pairs of bundles.

\section{The cylinder}

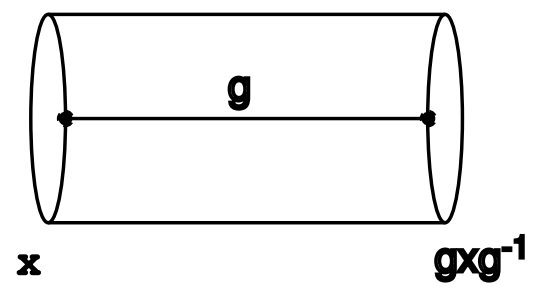

Figure 24: Pointed bundle over the cylinder

Consider the cylinder $I \times S^{1}$. If $Q \rightarrow I \times S^{1}$ is a bundle with basepoints, then we can determine group elements $g, x \in G$ where $g$ is the parallel transport from one end to the other, and $x$ is the 
holonomy around the left end. The holonomy around the right end is $g x g^{-1}$ (Figure 24). Now consider the gluing
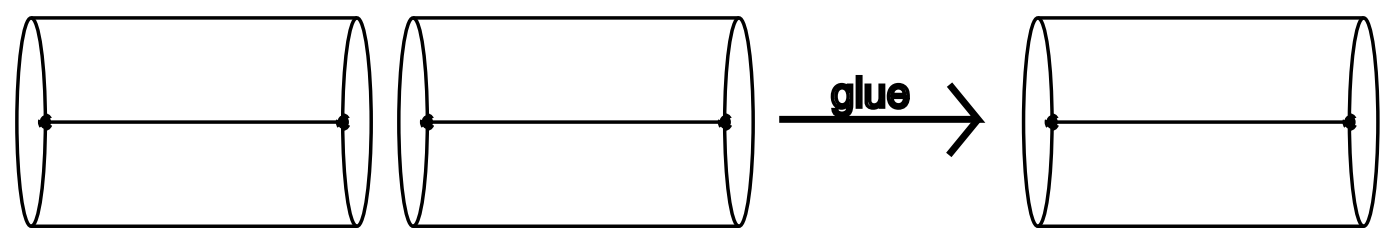

Figure 25: Gluing pointed cylinders

If we have bundles $Q_{1}, Q_{2}$ over the cylinders on the left, we can glue if the holonomies in middle agree. So if $Q_{i}$ is given by group elements $\left\langle x_{i}, g_{i}\right\rangle$, then we can glue if and only if $x_{2}=g_{1} x_{1} g_{1}^{-1}$.

We picture the bundle $\langle x, g\rangle$ as an arrow

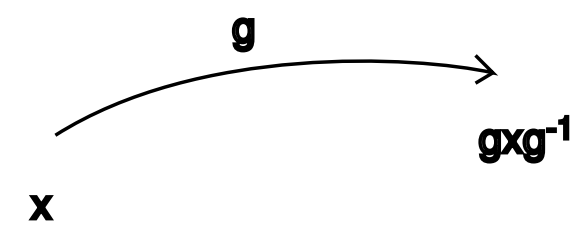

Figure 26: Schematic representation of a pointed bundle over the pointed cylinder

So the set of $(\# G)^{2}$ equivalence classes of bundles over $I \times S^{1}$ (always with basepoints) is the set of arrows in the picture:
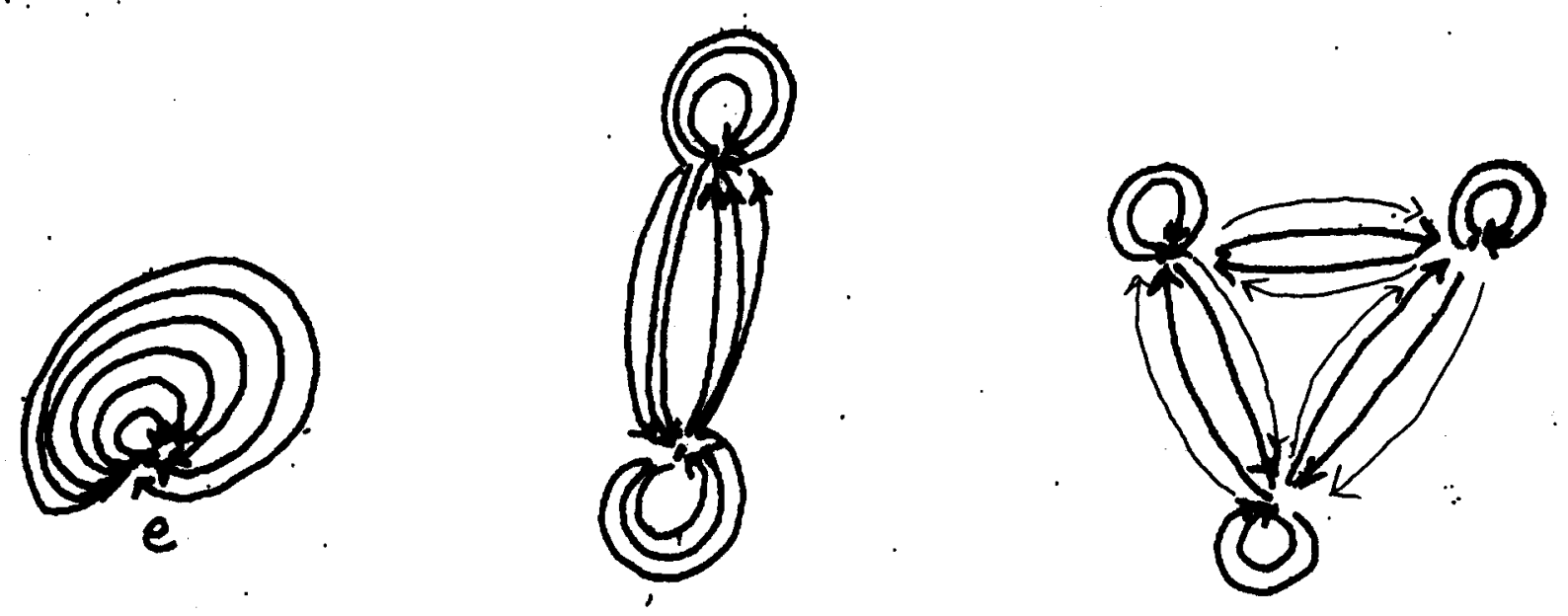

Figure 27: Equivalence classes of pointed bundles over the cylinder

(This is a picture for the symmetric group $S_{3}$, in fact.) Note that at the identity $e$ sits a copy of $G$, and more generally the set of arrows from $x$ to $x$ is the centralizer subgroup $C_{x} \subset G$. This is the subgroup of elements of $G$ which commute with $x$. The set of all arrows is a groupoid.

Exercise 28 Draw this picture for an abelian group $G$. 


\section{Quantization}

The measure we use on $\overline{\mathcal{C}_{Y}^{\prime}}$ for any $Y$ is still (19), but now understood with basepoints. If $Y$ has no closed components, then there are no nontrivial automorphisms, and the measure is identically 1.

The generalization of (22) is

$$
E(Y)=L^{2}\left(\overline{\mathcal{C}_{Y}^{\prime}}, \mu_{Y}\right)
$$

for any compact surface $Y$ (with basepoints).

Let's first consider the cylinder. We've already seen that $\overline{\mathcal{C}_{I \times S}^{\prime}}$ is a groupoid. This differs from a group in that composition is not always possible. Now, the multiplication

$$
m: H \times H \longrightarrow H
$$

on an arbitrary group $H$ determines by duality a coalgebra structure

$$
\Delta: \mathcal{F}(H) \longrightarrow \mathcal{F}(H \times H) \cong \mathcal{F}(H) \otimes \mathcal{F}(H)
$$

on the vector space of functions $\mathcal{F}(H)$ on the group. (The last isomorphism needs to be thought about if $H$ is not a finite group.) The same holds for our groupoid of fields on the cylinder.

Proposition. Let $A=E\left(I \times S^{1}\right)$. Then $A$ is a coalgebra. That is, there is a comultiplication

$$
\Delta: A \rightarrow A \otimes A
$$

and a counit

$$
\varepsilon: A \rightarrow \mathbb{C}
$$

There is also a "compatible" inner product, the $L^{2}$ inner product. We give the formulas, writing an equivalence class of bundles on $I \times S^{1}$ as a pair of group elements $\langle g, x\rangle$. Suppose $a \in A$. So $a: \overline{\mathcal{C}_{I \times S^{1}}^{\prime}} \rightarrow \mathbb{C}$. An element of $A \otimes A$ is a function on $\overline{\overline{\mathcal{C}}_{I \times S^{1}}^{\prime}} \times \overline{\mathcal{C}_{I \times S^{1}}^{\prime}}$. The coproduct is

$$
\begin{aligned}
\Delta a\left(\left\langle x_{1}, g_{1}\right\rangle,\left\langle x_{2}, g_{2}\right\rangle\right) & = \begin{cases}a\left(\left\langle x_{1}, g_{2} g_{1}\right\rangle\right. & \text { if } x_{2}=g_{1} x_{1} g_{1}^{-1} \\
0, & \text { if } x_{2} \neq g_{1} x_{1} g_{1}^{-1} .\end{cases} \\
\varepsilon(a) & =\sum_{x} a(\langle x, e\rangle)
\end{aligned}
$$

\section{Gluing Laws}

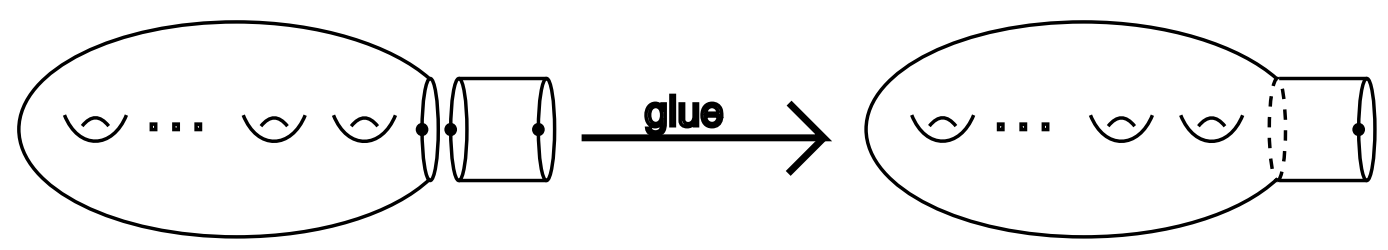

Figure 28: Gluing a pointed cylinder onto a pointed surface 
Now consider an arbitrary compact surface $Y$. By gluing a cylinder onto the boundary (Figure 28) we obtain an "action" of the groupoid $\overline{\mathcal{C}_{I \times S^{1}}^{\prime}}$ on $\overline{\mathcal{C}_{Y}^{\prime}}$. In the quantum picture this translates to

Proposition. $E(Y)$ is a unitary comodule for the coalgebra $A$.

A comodule is a corepresentation, dual to a representation of an algebra. Finally, the gluing law states that in the picture

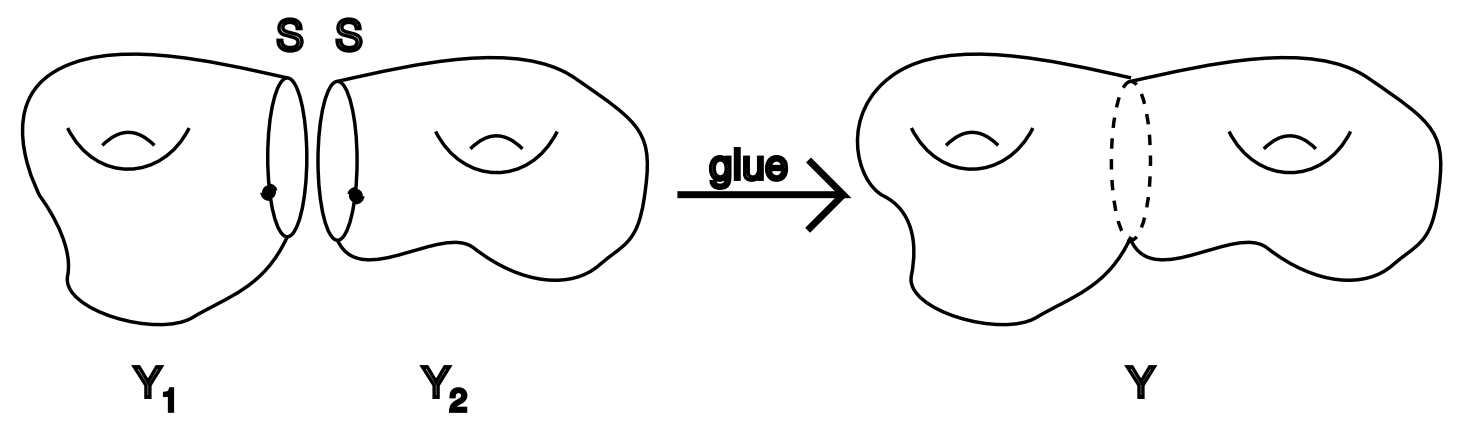

Figure 29: Gluing pointed surfaces

we have

$$
E(Y) \cong \frac{1}{\# G} E\left(Y_{1}\right) \otimes_{A} E\left(Y_{2}\right)
$$

Here ' $\nabla_{A}$ ' is the cotensor product, which is dual to the tensor product of modules of algebras. The factor $1 / \# G$ is for the inner product.

\section{Conformal Blocks}

This picture can be made more concrete, and so related to standard notions in conformal field theory. Briefly, the coalgebra $A$ is semisimple, so there is a finite number of irreducible corepresentations up to isomorphism, say $E_{1}, \ldots, E_{N}$. Thus for any compact surface $Y$ we can decompose the comodule (corepresentation) $E(Y)$ into irreducibles:

$$
E(Y) \cong \bigoplus_{\lambda} E(Y, \lambda) \otimes E_{\lambda}
$$

where now the $E(Y, \lambda)$ are vector spaces. The $\lambda$ which label the irreducible representations are called the labels, and we think of assigning the vector space $E(Y, \lambda)$ to the surface $Y$ with boundary labeled by $\lambda$. This is called a conformal block in the physics literature; Graeme Segal $[\mathrm{S}]$ uses the term modular functor. Whatever the language, the gluing law takes the form

$$
E(Y) \cong \bigoplus_{\lambda} \frac{\operatorname{dim} E_{\lambda}}{\# G} \cdot E\left(Y_{1}, \lambda\right) \otimes E\left(Y_{2}, \lambda\right)
$$

The factor refers to the inner product structure; it multiplies the inner product on the Hilbert space which follows. 
Exercise 29 Show that the definitions

$$
\begin{aligned}
\widetilde{E}\left(S^{1}\right) & =E\left(S^{1} \times S^{1}\right) \\
\widetilde{Z}_{Y} & =\operatorname{dim} E(Y)
\end{aligned}
$$

define a $1+1$ dimensional TQFT. The associated algebra is called the Verlinde algebra.

Exercise 30 By the axioms of TQFT, specifically the Functoriality Axiom, the symmetries of $S^{1} \times S^{1}$ act on $E\left(S^{1} \times S^{1}\right)$. This gives a representation of $S L_{2}(\mathbb{Z})$ on $E\left(S^{1} \times S^{1}\right)$. Compute this representation.

Exercise 31 Show that the factor in (35) is $S_{\lambda}^{1}$, where $S$ is the matrix $\left(\begin{array}{cc}0 & 1 \\ -1 & 0\end{array}\right)$ in $S L_{2}(\mathbb{Z}), 1$ is the trivial representation, and $\lambda$ is the given label $\lambda$. The matrix element $S_{\lambda}^{1}$ refers to the action of $S$ on $E\left(S^{1} \times S^{1}\right)$, as computed in Exercise 30 .

\section{Tensor Products}

I just mention that the vector space $E(P)$ of the pair of pants

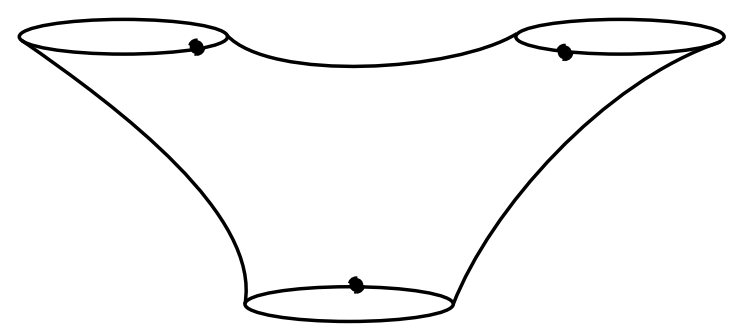

Figure 30: Pointed pair of pants

plays a special role. Namely it is an $(A \times A \times A)$-comodule since there are 3 boundary components. Then if $E_{1}$ and $E_{2}$ are $A$-comodules we can form a new $A$-module $E_{1} \otimes E_{2}$ by taking a sort of double cotensor product over $A$ :

$$
E_{1} \otimes E_{2}=E_{1} \otimes_{A} E(P) \otimes_{A} E_{2}
$$

This leads to a Hopf algebra structure on $A$, or more precisely to a Hopf algebra whose underlying coalgebra is isomorphic to $A$. Hopf algebras (and quasi-Hopf algebras) appeared in connection with finite groups in $[\mathrm{DPR}]$. From the classical action one first gets to the modular tensor categories, etc., which have been studied recently. The complete story is in in [F5]. 


\section{Lecture \#4: Classical $S_{2}$ Chern-Simons Theory}

We move now to the Chern-Simons gauge theory with gauge group $S U_{2}$, a topological theory in $2+1$ dimensions. The action is no longer trivial, and the space of equivalence classes of fields is not finite, nor even discrete. So new geometric and analytic ideas now enter, and indeed I would say that they are far from understood at this writing. In this lecture we discuss the classical theory; in the next lecture we turn to the quantum theory.

Classical mechanics and classical field theory have long been studied by mathematicians, certainly dating back to Newton's time. Over the years it has inspired many mathematical developments, notably the calculus of variations and symplectic geometry (corresponding to the Lagrangian and Hamiltonian pictures of the theory). More recently, classical gauge theory in physics was discovered to fit neatly into the independently developed mathematical theory of connections and curvature. So it is no surprise that the Chern-Simons lagrangian (see [CS] for the original reference in the mathematics literature) can be understood within our present-day mathematics. In fact, a careful study is illuminating, and I refer to my series of papers in progress [F1], [F2], and [F3] for a detailed development. A survey of these ideas appears in my lecture in the Colloquium connected to this Summer School [F4]. Here we outline some of the basic ideas for the $S U_{2}$ case.

\section{Fixed Data}

The dimension of the theory is $d=2$; this is a $2+1$ dimensional theory. Our gauge group is the Lie group

$$
G=S U_{2}=\left\{\left(\begin{array}{cc}
\alpha & \beta \\
-\bar{\beta} & \bar{\alpha}
\end{array}\right): \alpha, \beta \in \mathbb{C},|\alpha|^{2}+|\beta|^{2}=1\right\} .
$$

As a manifold $G$ is diffeomorphic to the 3 -sphere $S^{3}$. We also need to fix a bilinear form on the Lie algebra

$$
\mathfrak{g}=\mathfrak{s u}_{2}=\left\{\left(\begin{array}{cc}
i x & \alpha \\
-\bar{\alpha} & -i x
\end{array}\right): x \in \mathbb{R}, \alpha \in \mathbb{C}\right\} .
$$

This bilinear form is

$$
\langle a, b\rangle=\frac{-k}{8 \pi^{2}} \operatorname{Tr}(a b), \quad a, b \in \mathrm{SU}_{2},
$$

for some integer

$$
k=1,2, \ldots
$$

Note that (36) is invariant under conjugation. The reason for this normalization is the following. Let $\theta$ be the Maurer-Cartan 1-form on $S U_{2}$. If $g \in S U_{2}$ is written as a matrix, we write

$$
\theta=g^{-1} d g
$$

which is a matrix valued 1 -form, and the values are elements of $\mathfrak{s u}_{2}$, i.e., traceless skew-hermitian matrices. 
Exercise 32 Write $g=\left(\begin{array}{cc}\alpha & \beta \\ -\bar{\beta} & \bar{\alpha}\end{array}\right)$ and write a formula for $\theta$ in terms of $\alpha, \beta$.

Our normalization of (36) is chosen so that

$$
\int_{S U_{2}} \frac{k}{24 \pi^{2}} \operatorname{Tr}\left(\theta^{3}\right) \quad \text { is an integer. }
$$

Exercise 33 Verify (37).

\section{Fields}

Our spacetimes are compact oriented 3 -manifolds $X$. The fields are gauge fields or connections:

$$
\mathcal{C}_{X}=\left\{S U_{2} \text { connections } \Theta \text { over } X\right\}
$$

What is a connection? First of all it lives on a principal $G$ bundle $P \rightarrow X$. So $P$ is a manifold on which $G$ acts (on the right) freely with quotient $X$. This is the same definition as in Lecture \#2, where $G$ was a finite group. But now $S U_{2}$ has no nontrivial topology in dimensions 0 and 1 , and some elementary topology proves that there exists an isomorphism $P \cong X \times G$, where $X \times G$ is the trivial $G$ bundle with $G$ action

$$
\begin{gathered}
(X \times G) \times G \longrightarrow(X \times G) \\
\langle x, g\rangle \times h \longmapsto\langle x, g h\rangle .
\end{gathered}
$$

Another way to say this is to say that there exists a section

$$
p: X \rightarrow P
$$

of the principal bundle $\pi: P \rightarrow X$; that is, $\pi \circ p=i d_{X}$.

Since all $S U_{2}$ bundles are trivial, we might think of fixing once and for all $P=X \times G$. However, as we saw in the theory with finite gauge group, this does not exhibit all of the symmetry when we glue bundles. Recall that an isomorphism of bundles is a map

$$
\varphi: P^{\prime} \rightarrow P
$$

such that

$$
\varphi\left(p^{\prime} \cdot g\right)=\varphi\left(p^{\prime}\right) g
$$

for all $g \in G, p^{\prime} \in P^{\prime}$ and $\varphi$ induces the identity map on the quotients $P^{\prime} / G=P / G=X$. If $p^{\prime}=p$ then $\varphi$ is called a gauge transformation. We denote the group of gauge transformations by $\mathcal{G}_{P}$.

Exercise 34 Show that the group of gauge transformations of the trivial bundles $X \times G$ can be identified with the group of maps $\operatorname{Map}(X, G)$ operating by left multiplication.

Exercise 35 


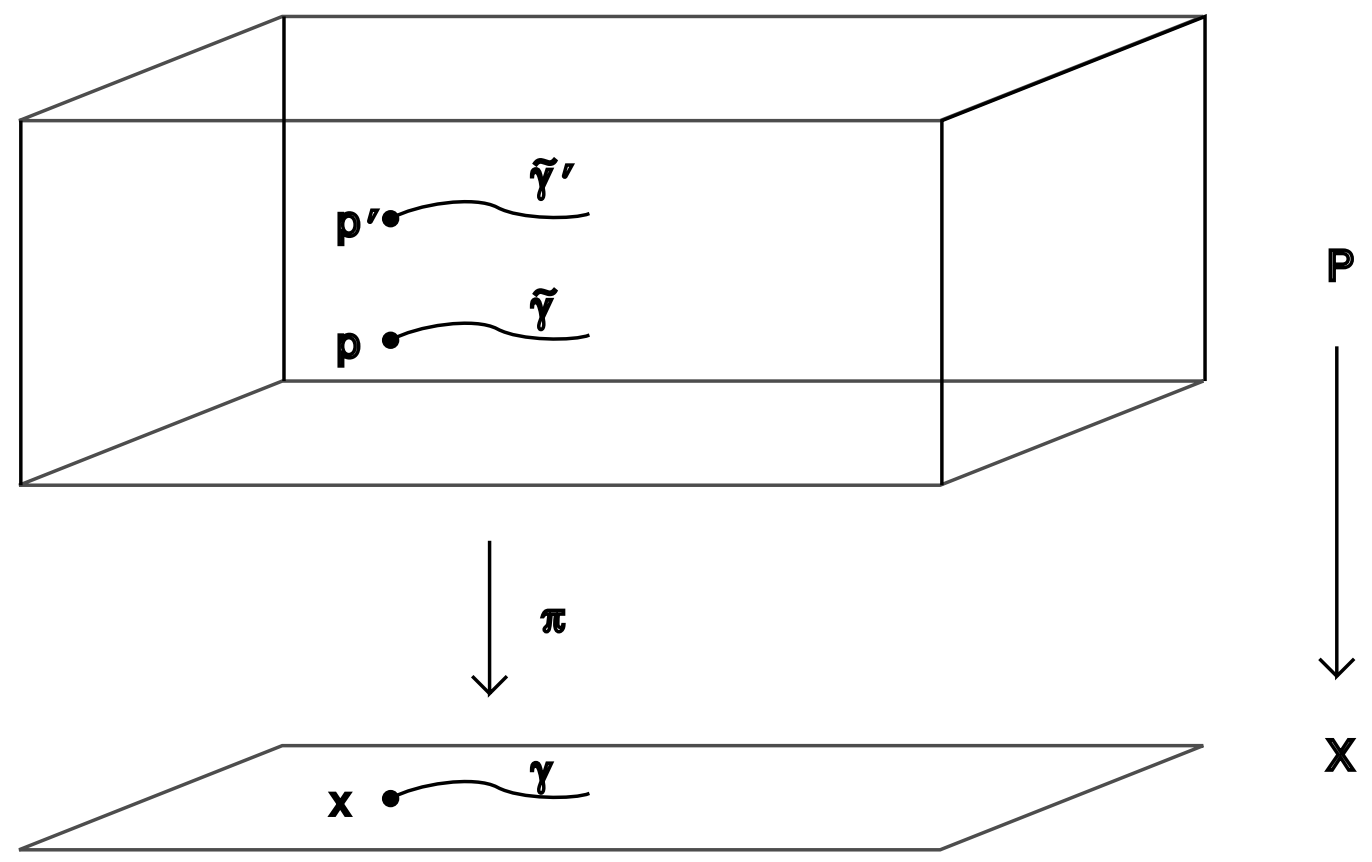

Figure 31: Horizontal lifts in a principal bundle with connection

a) If $X$ is a closed, oriented, and connected 3-manifold, use the 3-form (37) to show how to distinguish different homotopy classes of maps $X \rightarrow G$, i.e., different components of $\operatorname{Map}(X, G)$.

b) The set of components $\pi_{0} \mathcal{G}$ of any group $\mathcal{G}$ has a natural group structure. Show that if $X$ is a closed, oriented, and connected 3-manifold, then

$$
\pi_{0} \operatorname{Map}(X, G) \cong \mathbb{Z}
$$

Geometrically, a connection $\Theta$ is a structure on $P$ which enables us to lift paths on $X$ in a $G$ invariant way. (Figure 31) That is, given a path $\gamma$ in $X$ starting at $x \in X$, and a point $p \in P_{x}$ in the fiber of $P$ over $x$, then there is a lifted path $\tilde{\gamma}$ in $P$ starting at $p$ so that $\pi(\tilde{\gamma})=\gamma$. Of course such paths exist; the connection tells us how to choose one. If $p^{\prime}=p \cdot g$ is another point of $P_{x}$, then the lifted path $\tilde{\gamma}^{\prime}$ starting at $p^{\prime}$ must equal $\tilde{\gamma} \circ g$.

We will return presently to the analytic expression for a connection.

\section{Chern-Simons Action on $S^{3}$}

As a warm-up we first look at a geometric definition of holonomy for circle bundles with connection. It suffices to consider the trivial bundle $S^{1} \times \mathbb{T} \stackrel{\pi}{\longrightarrow} S^{1}$. Here $S^{1}$ is the circle as a manifold and $\mathbb{T}$ is the circle group, also called $U_{1}$ :

$$
\mathbb{T}=\left\{e^{i \theta}: \theta \in \mathbb{R}\right\}=\{\lambda \in \mathbb{C}:|\lambda|=1\} .
$$




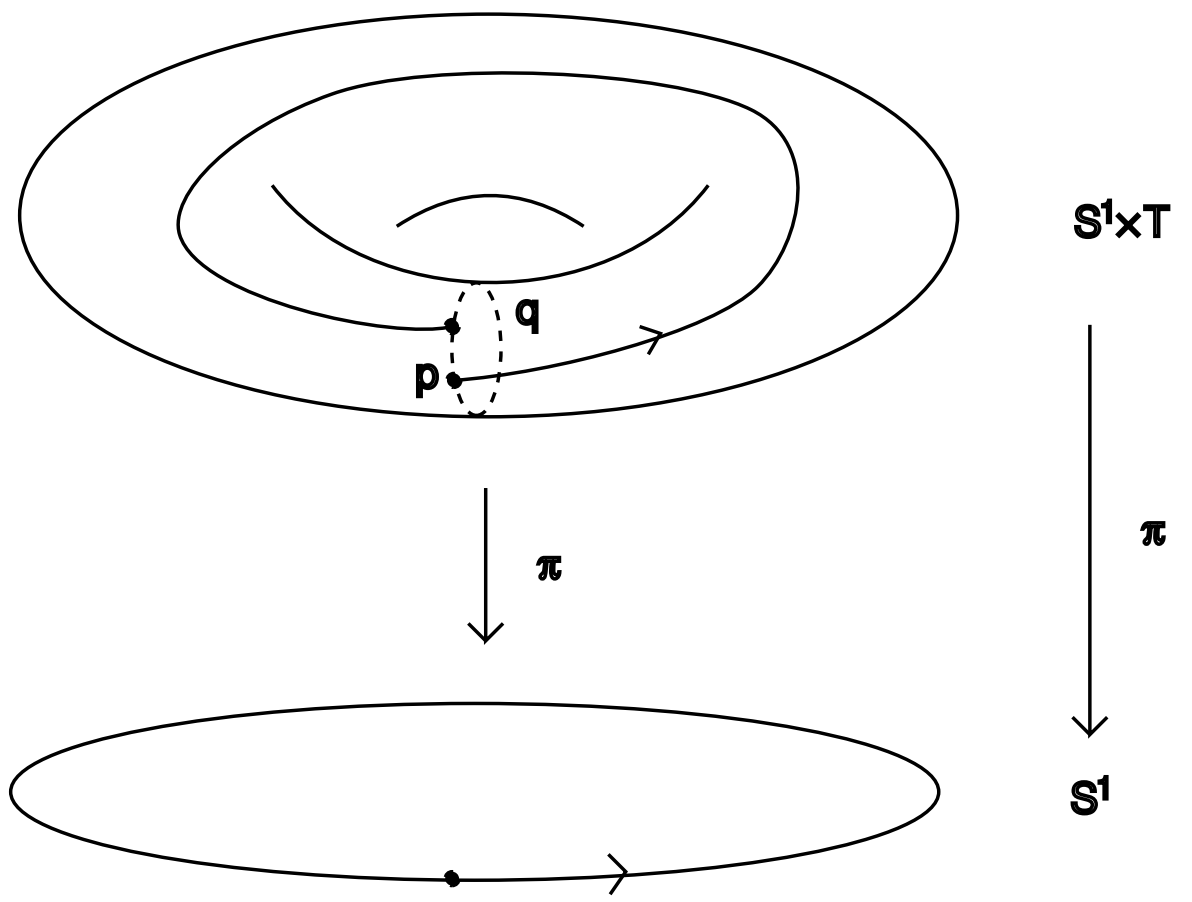

Figure 32: Holonomy of a connection on a $\mathbb{T}$ bundle over $S^{1}$

Now a connection on this bundle gives us a way to lift the base to a path in $S^{1} \times \mathbb{T}$, which is not necessarily closed, if we give an initial point $p$. Let $q$ be the terminal point. Now in the fiber over $x$, here identified with $\mathbb{T}$, we can measure the directed length from $p$ to $q$. Suppose the total length of $\mathbb{T}$ is 1 ; then this directed length $\ell$ is only well-defined up to addition of integers, since we can go around the circle any number of times to measure the directed length. So $e^{2 \pi i \ell} \in \mathbb{T}$ is well-defined, and this is the holonomy. (See Figure 32)

For an $S U_{2}$ connection over $S^{3}$ the Chern-Simons action is defined similarly. Consider the trivial bundle $P=S^{3} \times S U_{2} \stackrel{\pi}{\longrightarrow} S^{3}$ with a connection $\Theta$. Fix a point $x$ in $S^{3}$ and let $x^{\prime}$ be the antipodal point. Also, fix $p \in P_{x}$. Then lift all of the semi-great circles from $x$ to $x^{\prime}$ starting at $p$. (See Figure 33.) The lifted paths terminate in the fiber $P_{x^{\prime}}$ which we identify with $S U_{2}$. (Recall this is diffeomorphic to a 3 -sphere.) These terminal points fill out a 2 -sphere in $P_{x^{\prime}}$. In fact, the semi-great circles are parametrized by the equatorial $S^{2}$, so the terminal points comprise a map $\tau: S^{2} \rightarrow P_{x^{\prime}}$. Let $R$ be an oriented region in $P_{x^{\prime}}$ whose boundary is the image of $\tau$ (Figure 34). Then the Chern- 


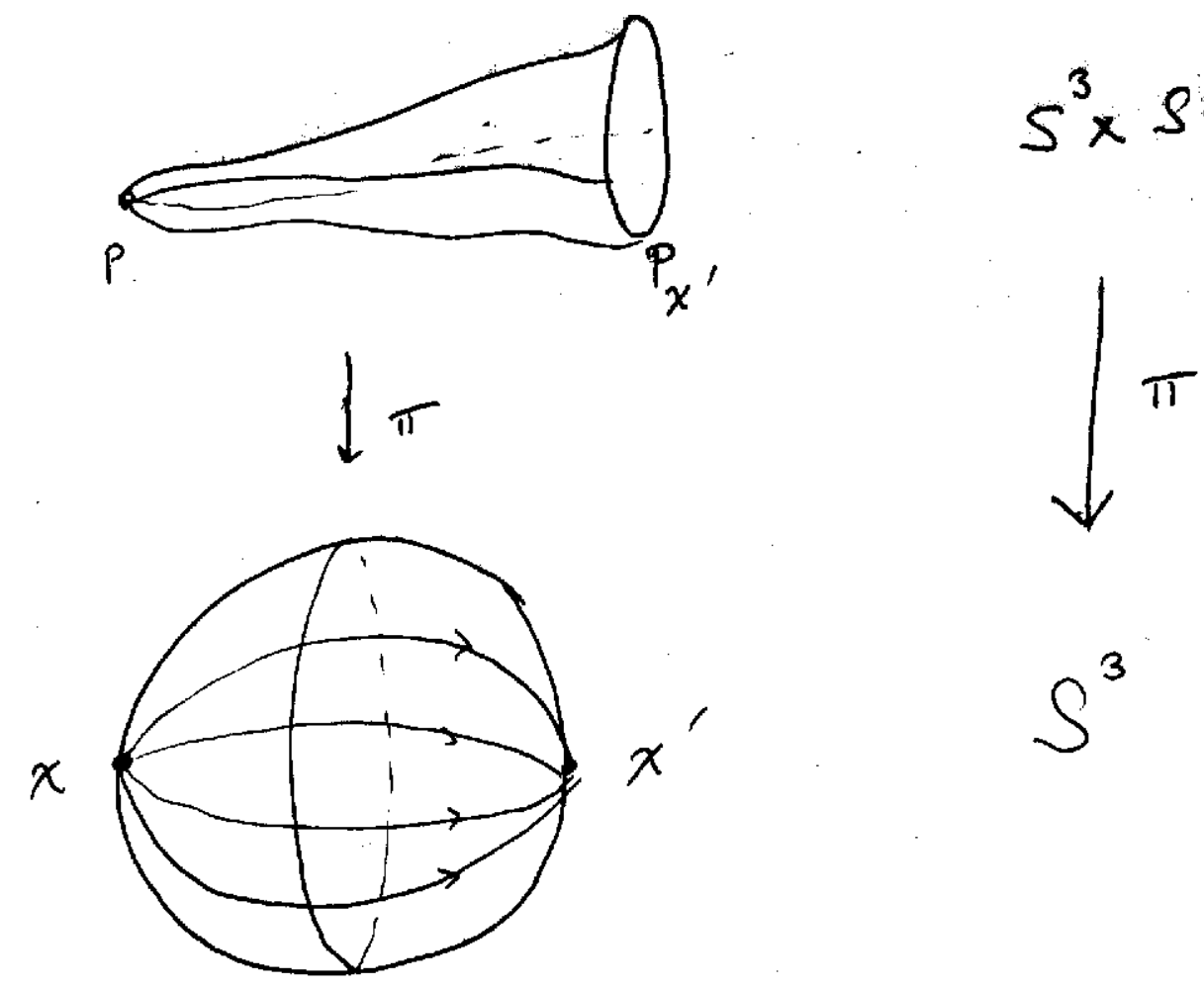

Figure 33: Holonomy of a connection on an $S U_{2}$ bundle over $S^{3}$

Simons action is

$$
S_{X}(\Theta)=\int_{R} \frac{k}{24 \pi^{2}} \operatorname{Tr}\left(\theta^{3}\right)
$$

Because of the condition (37), if we change the region $R$, then (38) changes by an integer. So the quantity

$$
e^{2 \pi i S_{X}(\Theta)} \in \mathbb{T}
$$

is well-defined. Notice that (38) is just the Wess-Zumino-Novikov-Witten action for the map $\tau$. Already we see a relationship between-Simons and Wess-Zumino-Novikov-Witten. We can think of this relationship as a transgression, which is not a sin, but rather a relationship in a fiber bundle between the geometry of the fiber and the geometry of the base (with a shift in dimension). Here the 2 dimensional geometry of the fiber is related to the 3 dimensional geometry of the base.

\section{Chern-Simons Action on closed spacetimes}

Now let $\Theta$ be a connection on a bundle $\pi: P \rightarrow X$, where $X$ is an arbitrary closed, oriented 3-manifold. We now need analytic formulas to define the Chern-Simons action. Rather than give an 


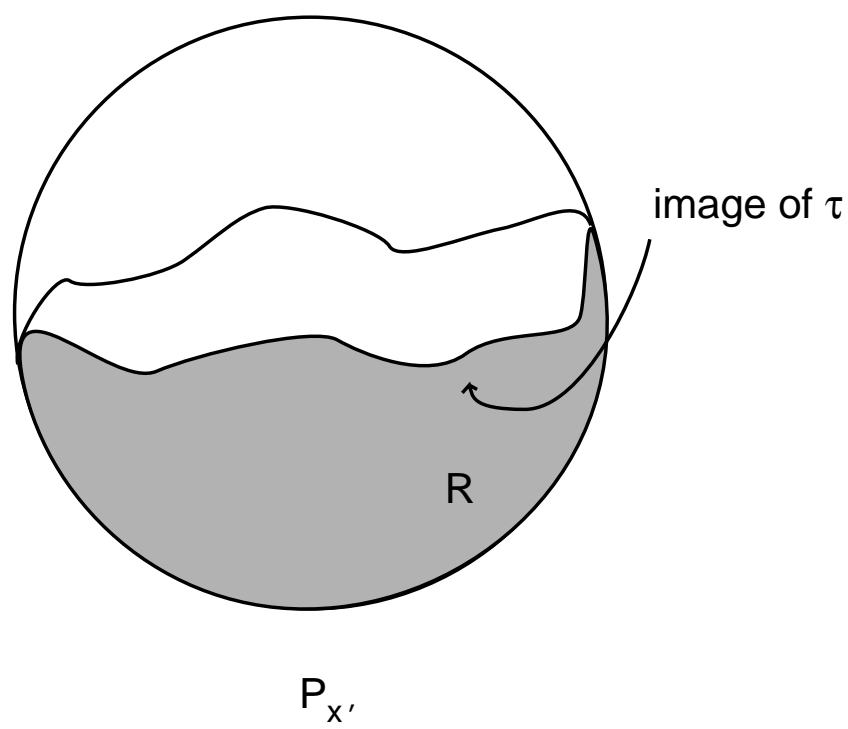

Figure 34: Bounding the image of $\tau$

abstract treatment of connections in general, which you can find in my paper [F1], let me express this in a form often found in the physics literature. Thus suppose $p: X \rightarrow P$ is a section of $P \stackrel{\pi}{\longrightarrow} X$, i.e., $\pi \circ p=i d_{X}$. Set

$$
p^{*} \Theta=A=A_{i} d x^{i}
$$

relative to local coordinates $x^{1}, x^{2}, x^{3}$ on $X$. Here $A_{i}=A_{i}(x)$ are functions on $X$ with values in the Lie algebra $\mathfrak{g}$. If $p^{\prime}$ is a different section, then we can write

$$
p^{\prime}=p \cdot g
$$

for some function $g: X \rightarrow G$. We have

$$
\begin{aligned}
p^{*} \Theta=A^{1} & =g^{-1} A g+g^{-1} d g \\
& =\left(g^{-1} A_{i} g+g^{-1} \frac{\partial g}{\partial x^{i}}\right) d x^{i}
\end{aligned}
$$

The curvature $\Omega$ is a $\mathfrak{g}$-valued 2 -form on $P$ constructed from the connection $\Theta$, which recall is a $\mathfrak{g}$-valued 1-form on $P$ :

$$
\Omega=d \Theta+\frac{1}{2}[\Theta \wedge \Theta]
$$

The formula on $X$, induced by a section $p: X \rightarrow P$, is perhaps more familiar:

$$
p^{*} \Omega=F=d A+A^{2}=\frac{1}{2} F_{i j} d x^{i} \wedge d x^{j}=\frac{1}{2}\left(\frac{\partial A_{j}}{\partial x^{i}}-\frac{\partial A_{i}}{\partial x^{j}}+\left[A_{i}, A_{j}\right]\right) d x^{i} \wedge d x^{j}
$$


Exercise 36 If $p^{\prime}$ is another section as in (39), show

$$
p^{\prime *} \Omega=F^{\prime}=g F g^{-1} .
$$

The Chern-Simons form $\alpha$ is a 3 -form which lives on $P$ :

$$
\alpha=\alpha(\Theta)=\langle\Theta \wedge \Omega\rangle-\frac{1}{6}\langle\Theta \wedge[\Theta \wedge \Theta]\rangle .
$$

Again, the form on the base may look more familiar:

$$
\begin{aligned}
p^{*} \alpha & =\frac{-k}{8 \pi^{2}} \operatorname{Tr}\left(A \wedge F-\frac{1}{3} A^{3}\right) \\
& =\frac{-k}{8 \pi^{2}} \operatorname{Tr}\left(\frac{1}{2} A_{i} F_{j k}-\frac{1}{3} A_{i} A_{j} A_{k}\right) \varepsilon^{i j k} d x^{1} \wedge d x^{2} \wedge d x^{3} .
\end{aligned}
$$

Exercise 37

a) Compute $d \alpha$ in case $X$ has dimension $\geq 4$.

b) Compute $\left(p^{\prime}\right)^{*} \alpha$ for the section (39) in terms of $p^{*} \alpha$.

Define an action which now depends on the section $p$ :

$$
S_{X}(\Theta, p)=\int_{X} p^{*} \alpha(\Theta) .
$$

We would like to get rid of the dependence on $p$, so we investigate how $S_{X}$ changes when we change $p$. Using Exercise 37b we see that for the section $p^{\prime}(39)$ :

$$
S_{X}\left(\Theta, p^{\prime}\right)=S_{X}(\Theta, p)+\int_{X} d\left\langle g^{-1} A g \wedge g^{-1} d g\right\rangle-\frac{1}{6} \int_{X}\left\langle g^{-1} d g \wedge\left[g^{-1} d g \wedge g^{-1} d g\right]\right\rangle .
$$

The second term vanishes by Stokes' Theorem, if $\partial X=0$. The third term can be rewritten as

$$
\int_{X} \frac{k}{24 \pi^{2}} \operatorname{Tr}\left(\left(g^{-1} d g\right)^{3}\right)
$$

If $X$ is closed, this is an integer by (37). So (40) is independent of $p$ up to an integer. Hence

$$
e^{2 \pi i S_{X}(\Theta)} \in \mathbb{T}, \quad X \text { closed, } \quad \Theta \in \mathcal{C}_{X},
$$

is well-defined.

Exercise 38 Check that if $\varphi: P^{\prime} \rightarrow P$ is an isomorphism, then

$$
e^{2 \pi i S_{X}\left(\varphi^{*} \Theta\right)}=e^{2 \pi i S_{X}(\Theta)}
$$

In particular, taking $P^{\prime}=P$ we see that the Chern-Simons action is gauge invariant.

Exercise 39 Check how the action behaves under orientation reversal and disjoint union.

Notice that our action does not quite fit the axioms of Lecture \#1, in particular (1). The value is not in $\mathbb{R}$ but rather in $\mathbb{R} / \mathbb{Z}$, or upon exponentiating, in $\mathbb{T}$. There is a more drastic modification of these axioms for manifolds with boundary. 


\section{Chern-Simons Action on spacetimes with boundary}

Suppose $X$ is a compact oriented 3-manifold, possibly with nonempty boundary $\partial X$. Let $\Theta$ be a connection over $X$. We would like to compute the action by (40), but now we find a more complicated dependence on the trivializing section $p$. Namely, (41) still holds since it is a local formula. But now the second term is (Stokes' Theorem):

$$
\begin{aligned}
\int_{X} d\left\langle g^{-1} A g \wedge g^{-1} d g\right\rangle & =\int_{\partial X}\left\langle g^{-1} A g \wedge g^{-1} d g\right\rangle \\
& =\int_{\partial X} \frac{-k}{8 \pi^{2}} \operatorname{Tr}\left(g^{-1} A g \wedge g^{-1} d g\right) \\
& =\int_{\partial X} \frac{-k}{8 \pi^{2}} \operatorname{Tr}\left(g^{-1} A_{i} \frac{\partial g}{\partial y^{j}}\right) \varepsilon^{i j} d y^{1} \wedge d y^{2}
\end{aligned}
$$

Also, by the usual arguments for the Wess-Zumino-Novikov-Witten term, the third term (42) just depends on the restriction $\partial g$ of $g$ to $\partial X$ - call it $W_{\partial X}(\partial g)$ - if we consider it up to integers. Again we see the Wess-Zumino-Novikov-Witten action in $1+1$ dimensions related to Chern-Simons in $2+1$. Set

$$
c_{\partial X}(a, g)=\exp \left(2 \pi i \int_{\partial X} \frac{-k}{8 \pi^{2}} \operatorname{Tr}\left(g^{-1} a g \wedge g^{-1} d g\right)+W_{\partial X}(g)\right)
$$

where $a$ is a 1 -form on $\partial X$ with values in $\mathfrak{g}$ and $g: \partial X \rightarrow G$. Then the preceding is summarized by

$$
e^{2 \pi i S_{X}(\Theta, p \cdot g)}=c\left(p^{*} \partial \Theta, g\right) e^{2 \pi i S_{X}(\Theta, p)} .
$$

Both sides are elements of $\mathbb{T}$, or if we like elements of unit norm in $\mathbb{C}$ - that is, complex numbers of norm one.

So how do we make sense of the action? It certainly is not independent of the trivializing section $p: X \rightarrow P$. But the variation depends on the boundary values $\partial p: \partial X \rightarrow \partial P$ of $p$ and the restriction $\partial \Theta$ of $\Theta$ to the boundary $\partial X$. Let $S_{\partial P}$ be the space of all sections of $\partial P \rightarrow \partial X$. Then set

$$
L_{\partial X, \partial \Theta}=L_{\partial \Theta}=\left\{f: S_{\partial P} \rightarrow \mathbb{C}: f(q \cdot g)=c\left(q^{*} \partial \Theta, g\right) \cdot f(q)\right\} \text { for all } g: \partial X \rightarrow G \text {. }
$$

In other words, $L_{\partial \Theta}$ is the set of functions from sections of $\partial P \rightarrow \partial X$ to $\mathbb{C}$ which transform according to (43). This is a one dimensional complex vector space with an inner product, i.e., a hermitian line.

Exercise 40 Verify this last assertion.

Then equation (43) states that the action makes sense as an element of this hermitian line (of unit norm):

$$
e^{2 \pi i S_{X}(\Theta)} \in L_{\partial \Theta}
$$

So again we must modify our axioms for an action. Now the action is exponentiated. For a closed spacetime it is an element of $\mathbb{T}$ (or $\mathbb{C}$ ). For a nonclosed spacetime it is an element of a complex line. 
The construction of this Chern-Simons line applies to any connection $\eta$ an a $G$ bundle $Q \rightarrow Y$ over a closed oriented 2-manifold $Y$-we obtain a line $L_{Y, \eta}=L_{\eta}$.

Exercise 41 Verify the following properties of the lines $L_{\eta}$.

a) (Functoriality) If $\psi: Q^{\prime} \rightarrow Q$ is a bundle isomorphism for bundles $Q^{\prime}, Q$ over $Y$, and $\eta$ is a connection on $Y$, then there is an induced isometry

$$
\psi_{*}: L_{\psi^{*} \eta} \rightarrow L_{\eta}
$$

b) (Orientation) There is an isometry

$$
L_{-Y, \eta} \cong \overline{L_{Y, \eta}} .
$$

c) (Disjoint Union) There is an isometry

$$
L_{\eta_{1} \sqcup \eta_{2}} \cong L_{\eta_{1}} \otimes L_{\eta_{2}}
$$

if $\eta_{1}, \eta_{2}$ are fields (connections) on $Y_{1}, Y_{2}$.

Exercise 42 State and derive analogous properties of the Chern-Simons action. These should be analogous to the corresponding axioms in Lecture \#1.

\section{Gluing Law}

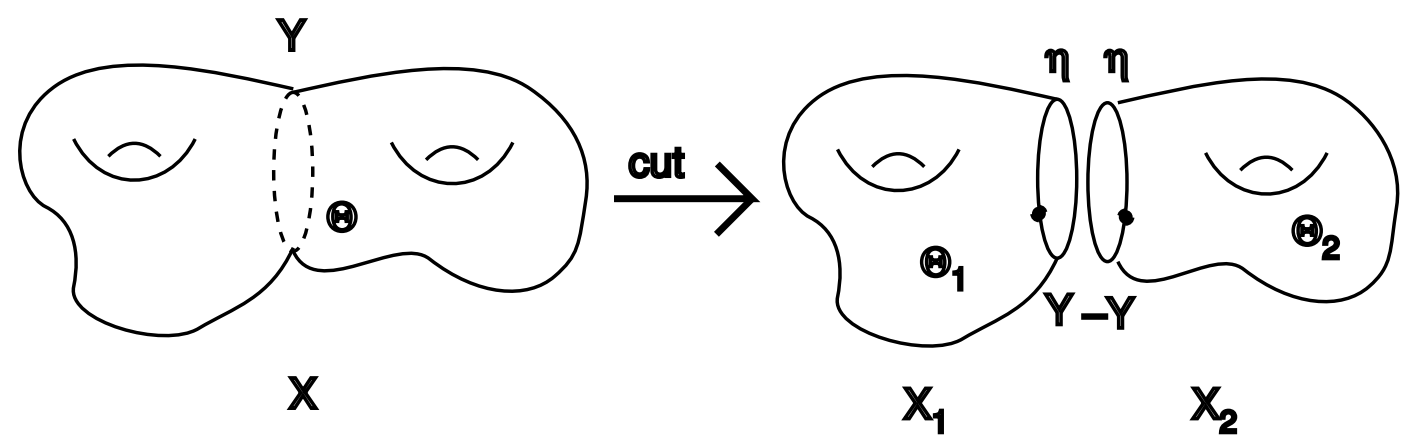

Figure 35: Gluing connections

The locality of a usual sort of action is the gluing law (6), (7). In Chern-Simons this gets modified to accommodate the different sort of action that we have. Also, because of the exponential it looks like a multiplicativity property rather than an additivity property. Figure 35 illustrates a typical gluing. A closed 3-manifold $X$ is cut along $Y$ and a connection $\Theta$ cut into two connections $\Theta_{1}, \Theta_{2}$ which both restrict to $\eta$ on $Y$. Then

$$
e^{2 \pi i S_{X_{1}}\left(\Theta_{1}\right)} \in L_{Y, \eta}, \quad e^{2 \pi i S_{X_{2}}\left(\Theta_{2}\right)} \in L_{-Y, \eta} \cong \overline{L_{Y, \eta}} .
$$


The gluing axiom is

$$
e^{2 \pi i S_{X}(\Theta)}=\left(e^{2 \pi i S_{X_{1}}\left(\Theta_{1}\right)}, e^{2 \pi i S_{X_{2}}\left(\Theta_{2}\right)}\right)
$$

where the inner product takes place in $L_{Y, \eta}$.

Exercise 43 Demonstrate (45).

\section{Classical Solutions}

We leave as an exercise the derivation of the variation of the action

$$
\delta S_{X}(\Theta)=2 \int_{X} \frac{-k}{8 \pi^{2}} \operatorname{Tr}(F \wedge \delta A)
$$

and the resultant Euler-Lagrange equations for the classical solutions:

$$
F=0
$$

(If $\partial X \neq \emptyset$ then we must assume that $\Theta$ is fixed on $\partial X$.) So a solution is a flat connection, i.e., a connection with zero curvature. Let

$$
\mathcal{M}_{X}=\text { space of flat connections on } X \text { up to equivalence. }
$$

As with the finite group case in Lecture \#2 (see (18)), where all connections are flat, it is true that

$$
\mathcal{M}_{X} \simeq \operatorname{Hom}\left(\pi_{1} X, G\right) / G
$$

This may be a finite set (see the Brieskorn spheres in Lecture \#5) or it may have continuous parameters.

\section{Hamiltonian Theory}

The Hamiltonian theory concerns spacetimes which are products of space and time, or better paths of fields in a space $Y$. Thus we study $X=[0, \infty) \times Y$. (The fact that this particular $X$ is noncompact won't bother us.) Fix a $G$ bundle $Q \rightarrow Y$, which is of course trivializable. Let

$$
\mathcal{A}_{Q}=\text { space of connections on } Q \text {. }
$$

Then up to gauge equivalence any connection on $[0, \infty) \times Q \rightarrow[0, \infty) \times Y$ is a path in $\mathcal{A}_{Q}$, as we see using the "temporal gauge". We want to see what the action is on such paths, but now on closed paths. 
Let $\eta_{t}, 0 \leq t \leq 1$, be a path of connections on $Q$. We view this as a connection $\boldsymbol{\eta}$ on the product bundle $[0,1] \times Q \rightarrow[0,1] \times Y$ which has no $d t$ component, i.e., in the temporal gauge. So we can consider the action $e^{2 \pi i S_{[0,1] \times Y}(\boldsymbol{\eta})}$. It is an element of the line attached to the boundary connections $\eta_{0} \sqcup \eta_{1}$. Because the orientation on the left of the cylinder is opposite the orientation on the right,

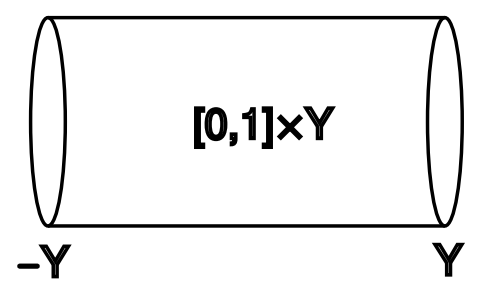

Figure $36:[0,1] \times Y$

the action is a linear map

$$
e^{2 \pi i S_{[0,1] \times Y}(\boldsymbol{\eta})}: L_{\eta_{0}} \rightarrow L_{\eta_{1}} .
$$

We picture this in Figure 37. The lines $L_{\eta}$ fit together into a complex line bundle

$$
L_{Q} \rightarrow \mathcal{A}_{Q}
$$

and the action gives us a way of lifting paths to act on $L_{Q}$. The gluing law for the action implies that these maps compose properly when we glue paths together (Figure 38). One can show that this means that (47) is the parallel transport of a connection on the bundle (48). This is the link between the Lagrangian and Hamiltonian Theories. One computes the curvature of this connection to be

$$
\omega\left(\delta_{1} A, \delta_{2} A\right)=-2 \int_{Y} \frac{-k}{8 \pi^{2}} \operatorname{Tr}\left(\delta_{1} A \wedge \delta_{2} A\right)
$$

where $\delta_{i} A$ are infinitesimal variations of the gauge field, i.e., tangent vectors in $\mathcal{A}_{Q}$. This curvature is a symplectic form on $\mathcal{A}_{Q}$.

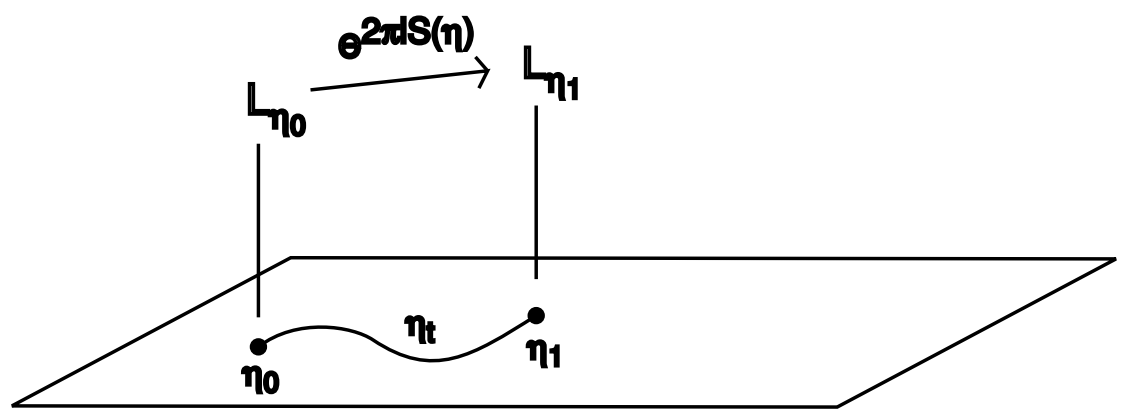

Figure 37: The action as parallel transport 


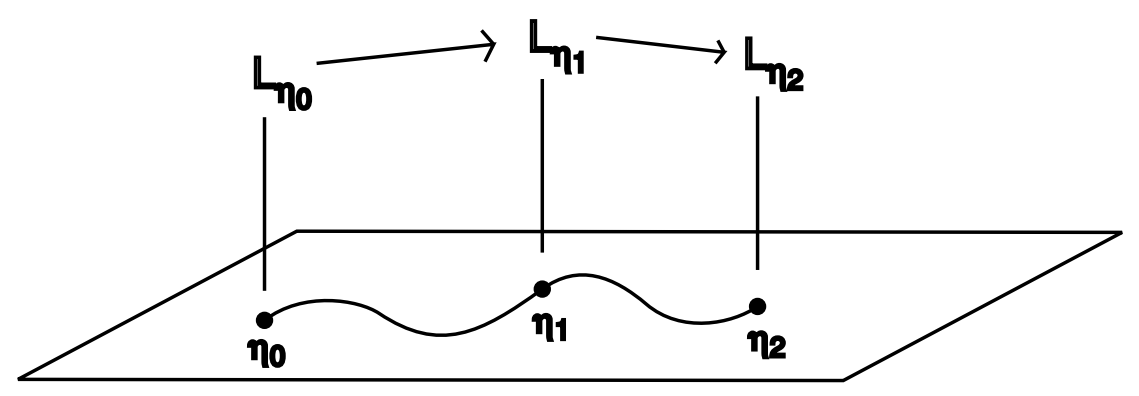

Figure 38: Composing paths and parallel transports

Again: The action on a path in the space of fields on space is the parallel transport for a connection on that space of fields. If the lagrangian is nondegenerate, its curvature is a symplectic form. Many mathematical treatments of Hamiltonian mechanics begin with this symplectic structure. Here we derive it from a Lagrangian theory. Also, (44) says that the action of gauge transformations $\mathcal{G}_{Q}$ on $\mathcal{A}_{Q}$ lifts to the line bundle $L_{Q}$. This lift is also constructed directly from the Chern-Simons action. So not only does the Hamiltonian Theory involve a symplectic manifold $\mathcal{A}_{Q}$ (phase space), but the action produces a line bundle $L_{Q} \rightarrow \mathcal{A}_{Q}$ with a connection whose curvature is the symplectic form. And, as mentioned, the gauge transformations $\mathcal{G}_{Q}$ lift to act on $L_{Q}$.

From this lifted action we can compute a momentum map for the action of $\mathcal{G}_{Q}$. Phase space reduction consists of restricting to the zero momentum subspace and then dividing out by $\mathcal{G}_{Q}$. Geometrically, the zero momentum space is the subspace of $\mathcal{A}_{Q}$ over which the connection on $L_{Q}$ drops to the quotient $\mathcal{A}_{Q} / \mathcal{G}_{Q}$. This is the space of flat connections, and so we obtain a quotient line bundle with connections

$$
L_{Y} \rightarrow \mathcal{M}_{Y}
$$

Here $\mathcal{M}_{Y}$ is the set of flat connections on $Y$ up to equivalence. This Chern-Simons line bundle and connection enters the quantization of the theory.

This construction of the line bundle appears in [RSW].

\section{Beyond the Hamiltonian Theory}

Recall from Lecture \#3 that we obtain a more intricate algebraic structure by considering (in that simple quantum theory) surfaces with boundary. it is natural to ask whether in this classical theory, and ultimately in its quantized version, one can profitably deal with surfaces with boundary. The answer is yes, though the story which develops is quite complicated. It involves strange algebraic objects called gerbes. We avoided them in Lecture \#3 by introducing basepoints; that can be done here as well $[\mathrm{F} 1, \S 4-\S 5]$. The full story will be presented in [F2] and [F3]. There we will finally understand the classical Chern-Simons action (for arbitrary gauge group) as the integral of the lagrangian over spacetime, just as ordinary actions, such as the harmonic map action(2), are integrals of a lagrangian over spacetime. Furthermore, one dimension lower we see the Chern-Simons line as the integral of the lagrangian over space. And this continues to lower dimensions and more 
complicated integrations. (See [F4] for a survey.) But in these topological theories the lagrangian and the integral are somewhat exotic. 


\section{Lecture \#5: Quantum $S U_{2}$ Chern-Simons Theory: Computer Calculations}

Although present-day mathematics is well-suited to describe classical field theory, and the ChernSimons theory in particular, the quantum field theory most likely requires new mathematical ideas. Certainly there is a large literature on the mathematics of quantum field theory, and there is much nontrivial progress towards understanding many aspects. But I think it is fair to say that the mechanism of quantization is still by and large a mystery. There is plenty of evidence to suggest that quantum field theory will lead to new developments in mathematics; indeed, it already has. Certainly mathematics has also contributed to quantum field theory as well. But, as I say, I believe there is more mystery here than light.

In this final lecture we abandon our theoretical tools and turn to computations in the quantum Chern-Simons theory. This is joint work with Bob Gompf, and is based on an influential paper of Witten [W1]. Witten considers the Chern-Simons theory for $S U_{2}$ at "level" $k$, as discussed in the previous lecture. (He also treats other gauge groups, but we stick to $S U_{2}$.) He does two things:

(i) Gives a prescription for computing the exact path integral $Z_{X}(k)$, depending on the level $k$;

(ii) Gives an asymptotic formula for $Z_{X}(k)$ as $k \rightarrow \infty$ by standard perturbation theory techniques.

The exact solution in (i) is, I believe, quite rare in quantum field theory; usually one only has the perturbation theory (ii). The asymptotic formula (see (53) below) involves more or less standard topological invariants of the closed oriented 3-manifold $X$ and representations of its fundamental group. Witten asserts that the exact formula for $Z_{X}(k)$ is a topological invariant, and this has been borne out by the work of Reshetikhin/Turaev [RT], Walker [Wa] and others. They start with complicated algebraic data and construct the exact solution. Recall that this is the reverse of what we did in Lecture \#3. From the special properties of the algebraic data they can prove the properties of quantum field theory (functoriality, gluing, etc.). In particular, they prove that $Z_{X}(k)$ is a topological invariant. While this is very important work, and an important step towards understanding Chern-Simons theory, it should be pointed out that:

(i) This work never mentions the Chern-Simons action.

(ii) Locality is not immediately apparent from this algebraic data. Better, I don't know of a notion of "locality" in their algebraic data which would suggest why one gets out a field theory.

(iii) They do not discuss the perturbative expansion, and it is not at all clear how the topological invariants involved in that expansion (see (53)) emerge from this algebraic data.

I only mean these comments to indicate that there is a long way to go towards understanding locality and quantum field theory in a manner usable in a variety of geometric and topological problems. They are not at all a criticism of this beautiful work, which is an important step towards a more complete understanding these theories.

We do computations of $Z_{X}(k)$ for some relatively simple 3 -manifolds $X$-lens spaces $X=L(p, q)$ 
and Brieskorn spheres $X=\sum(p, q, r)$. We will see the desired properties of the exact solution in our computations, and we will also see that the partition function is a nontrivial effective topological invariant, at least in these simple examples. We will also compute $Z_{X}(k)$ for large values of $k$ and compare with the asymptotic formula predicted by perturbation theory. We find excellent numerical agreement. For lens spaces this is now understood in analytic terms (i.e., proved!), but for Brieskorn spheres the analytic results are only qualitative. ${ }^{1}$ (See the theses of Lisa Jeffrey [J] and Stavros Garoufalidis [G].) The agreement between the exact solution and the perturbation theory, though expected from formal arguments, is another mystery about the path integral which we don't really understand, and which hints at new geometric structures.

Another discussion of these results from a physics perspective is contained in [FG2]. The complete results are contained in [FG1]. Since these references should be fairly accessible and readily available, our discussion here is brief.

\section{Exact Solution}

Let $e^{2 \pi i S_{X}(\Theta)}$ denote the Chern-Simons action constructed in the last lecture for $k=1$; then the action for arbitrary $k$ is $e^{2 \pi i k S_{X}(\Theta)}$. An important point to remember is this: We do not have measures available on the space of fields, so cannot quantize directly using measure theory as we did in Lecture \#2. Of course, we can assume the existence of a measure $d \mu_{X}$ on the space of fields, assume that the measure has all the good properties we need, and write a formal path integral

$$
Z_{X}(k)=\int_{\overline{\mathcal{C}_{X}}} e^{2 \pi i k S_{X}(\Theta)} d \mu_{X}(\Theta), \quad X^{3} \text { closed }
$$

and suppose that we have a map

$$
Y \rightsquigarrow E(Y), \quad Y^{2} \text { closed, }
$$

from closed 2-manifolds to finite dimensional Hilbert spaces such that these maps satisfy the axioms of Lecture \#1. (In fact, there is an anomaly which enters, which leads to certain central extensions and to the requirement that our spaces and spacetimes have a little more structure. We refer to Witten's paper [W1] for this and for more about the entire theory.) We also have the path integral over 3-manifolds with boundary, and we assume that we also have the refined theory involving surfaces with boundary as discussed in Lecture \#3.

Now, of course, Witten does not merely assume all of this and go on. There are definite criteria which indicate whether a quantum theory makes sense, and what modifications may have to be made (e.g., anomalies). Also, the procedure for producing the quantum Hilbert spaces (49) is well-studied - it is usually called canonical quantization and is the Hamiltonian picture of quantum theory. In this case Witten also appeals to geometric quantization for the construction of the quantum Hilbert space. There is much mathematical work towards justifying this construction (for example $[\mathrm{ADW}],[\mathrm{H}])$. Further, Witten noticed that the construction fits into conformal field theory,

\footnotetext{
${ }^{1}$ As we write Lev Rozansky is making great strides towards an analytic understanding.
} 
more specifically rational conformal field theory, and so he was able to borrow results from there to compute with the $E(Y)$. This is also one entree into the theory (discussed in Lecture \#3) involving surfaces with boundary. In any case, we simply observe here that the quantum Chern-Simons theory in $2+1$ dimensions is related to the quantum Wess-Zumino-Novikov-Witten (topological) theory in $1+1$ dimensions, just as the corresponding classical theories are related.

Just a word about the quantization. Recall the line bundle with connection

$$
L_{Y} \rightarrow \mathcal{M}_{Y}
$$

over the space of flat connections on $Y$. If $Y$ is given a complex structure, then $\mathcal{M}_{Y}$ and $L_{Y}$ inherit a complex structure, and the quantization is

$$
E(Y)=\operatorname{Hol}\left(\mathcal{M}_{Y} ; L_{Y}\right)
$$

the space of holomorphic sections of (50). To see that this is well-defined we must check that it is independent of the complex structure. (In fact, only the underlying projective space is independent, since there is an anomaly.) This is like quantizing a classical system with variables $p, q$ by the Hilbert space $\operatorname{Hol}(q+p \sqrt{-1})$ of holomorphic functions of the complex variable $q+p \sqrt{-1}$. Other choices are possible and typical — for example, $L^{2}$ functions of $q$ or $L^{2}$ functions of $p$. One of the main problems in quantizing is to identify all of the different quantizations, at least projectively, and that is what the mathematical work referred to in the previous paragraph does for the Chern-Simons theory.

These spaces have led to a solution of some mathematical old problems about $\mathcal{M}_{Y}$, which are of interest in the theory of Riemann surfaces.

\section{The torus}

One can identify the vector space

$$
E\left(S^{1} \times S^{1}\right) \cong \mathbb{C}\left\{e_{1}, \ldots, e_{k+1}\right\}
$$

as the span of certain vectors $e_{1}, \ldots, e_{k+1}$. (This canonical basis can be deduced from the refined gluing laws analogous to those in Lecture \#3.) The axioms of topological field theory say that the orientation-preserving diffeomorphisms of $S^{1} \times S^{1}$ should act on $E\left(S^{1} \times S^{1}\right)$, and the action only depends on homotopy classes of diffeomorphisms (see Exercise 18). That is, $S L_{2}(\mathbb{Z})$ acts on $E\left(S^{1} \times S^{1}\right)$. From conformal field theory, and ultimately from the theory of Kač-Moody Lie algebras $[\mathrm{KM}]$, one concludes that the generators

$$
T=\left(\begin{array}{ll}
1 & 1 \\
0 & 1
\end{array}\right) \text { and } S=\left(\begin{array}{cc}
0 & -1 \\
1 & 0
\end{array}\right)
$$

act on $E\left(S^{1} \times S^{1}\right)$ via the matrices

$$
\widetilde{T}_{\beta}^{\alpha}=e^{2 \pi i\left(h_{\alpha}-c / 24\right)} \delta_{\beta}^{\alpha} \quad ; \quad \widetilde{S}_{\beta}^{\alpha}=\sqrt{\frac{2}{k+2}} \sin \left(\frac{\alpha \beta \pi}{k+2}\right)
$$


where

$$
c=\frac{3 k}{k+2} \quad ; \quad h_{\alpha}=\frac{\alpha^{2}-1}{4(k+1)} .
$$

These are the explicit formulas we use in our computations below.

Other considerations from conformal field theory show

$$
\operatorname{dim} E\left(S^{2}\right)=1
$$

\section{Surgery Formulas}

So canonical quantization, or rather the link between the path integral and canonical quantization, together with some results from conformal field theory and Kač-Moody Lie algebras (or better topological Wess-Zumino-Novikov-Witten Theory) are one part of the exact solution. One should note that this makes heavy use of symmetry. The other part of the exact solution we need is derived directly from the set of axioms (see Lecture \#1) that the path integral is meant to satisfy.

We need a starting point, and this is

$$
Z_{S^{1} \times S^{2}}=1
$$

which follows from (51) and (27). The other main tool is the Gluing Law(16), from which we derive the change of the path integral under surgery.

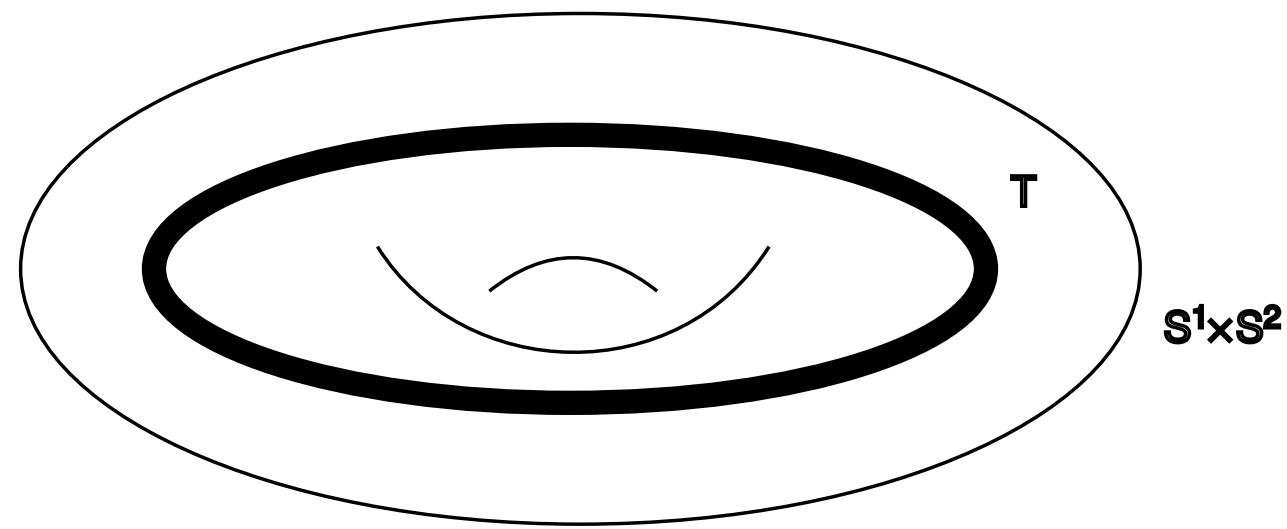

Figure 39: Writing $S^{1} \times S^{2}$ as the union of 2 solid tori

Let $T \subset S^{1} \times S^{2}$ be a solid torus as shown in Figure 39. The complement $X=\left(S^{1} \times S^{2}\right)-T$ is also a solid torus. We have

$$
S^{1} \times S^{2}=X \cup T
$$

glued along the common boundary $\partial T \cong S^{1} \times S^{1}$, which is an ordinary torus. This factors the partition function (52) into

$$
1=\left(Z_{X}, Z_{T}\right)_{E\left(S^{1} \times S^{1}\right)}
$$


If we glue $X$ and $T$ together by a diffeomorphism $f$ of $\partial T \cong S^{1} \times S^{1}$, then the Functoriality Axiom and the Gluing Law imply that

$$
Z_{X \cup_{f} T}=\left(Z_{X}, f_{*} Z_{T}\right)_{E\left(S^{1} \times S^{1}\right)}
$$

where $X \cup_{f} T$ is the new 3-manifold obtained by this surgery. If

$$
f=\left(\begin{array}{cc}
q & r \\
p & s
\end{array}\right) \in S L_{2} \mathbb{Z}
$$

then the resulting manifold is the lens space $L(p, q)$. A similar surgery on 3 parallel solid tori in $S^{1} \times S^{2}$ yields the Brieskorn sphere $\sum(p, q, r)$.

This is a sketch of the procedure given by Witten, and used by Gompf and me to compute some of the exact partition functions which we compute below.

\section{Perturbation Theory}

In this theory one should think of $k=1 / \hbar$, except that $\hbar$ is now "quantized" to be the reciprocal of an integer. So here the perturbative limit $\hbar \rightarrow 0$ is $k \rightarrow \infty$. The leading order behavior is determined around the critical points of the classical action, which are the (equivalence classes of) flat connections $A \in \mathcal{M}_{X}$ (see (46)). The perturbation theory then gives a formula in terms of topological invariants of the flat connection $A$. For simplicity we state it when the space $\mathcal{M}_{X}$ is finite and consists entirely of irreducible connections. This holds for $X=\sum(p, q, r)$, for example. The asymptotic formula is

$$
Z_{X}(k) \sim \frac{1}{2} e^{-3 \pi i / 4} \sum_{A \in \mathcal{M}_{X}} \sqrt{\tau_{X}(A)} e^{-2 \pi i I_{A} / 4} e^{2 \pi i(k+2) S_{X}(A)}
$$

where

$$
\begin{aligned}
T_{X}(A) & =\text { Reidemeister torsion of } X \text { with coefficients twisted by } A ; \\
I_{A} & =\text { a certain "spectral flow". It is an integer determined modulo } 8 .
\end{aligned}
$$

The Reidemeister torsion is a topological invariant introduced a long time ago to distinguish various lens spaces $[\mathrm{R}]$, [Fr]. It appears here in the form of analytic torsion, which was introduced by Ray and Singer [RS] as a certain combination of determinants of Laplace operators. The equality of the analytic torsion and Reidemeister torsion was established by Cheeger $[\mathrm{C}]$ and Müller $[\mathrm{M}]$. The spectral flow is a topological invariant introduced by Atiyah/Patodi/Singer [APS]. Note the shift $k \rightarrow k+2$ in the action, which occurs in various guises in different accounts of the Chern-Simons theory. We emphasize that (53) is derived (by Witten - see also [FG1,§1]) using standard Gaussian integrals and gauge fixing, together with a mathematical theorem which identifies a certain combination of determinants as the Reidemeister torsion. 


\section{The Computations}

We present only a few representative computations here. More complete results can be found in [FG1].

We gave surgery descriptions of the lens space $L(p, q)$ and the Brieskorn sphere $\Sigma(p, q, r)$ above. (Here $p, q$ and $p, q, r$ are pairwise relatively prime.) Now we give another description of these spaces simply to help the reader picture them. For the lens space, we start with the 3 -sphere $S^{3}$ thought of as the set of points in $\mathbb{C}^{2}=\{\langle z, w\rangle\}$ of unit norm. Let the cyclic group $\mathbb{Z} / p \mathbb{Z}$ act on $\mathbb{C}^{2}$ by letting the generator be the transformation

$$
\langle z, w\rangle \longmapsto\left\langle\omega z, \omega^{q} w\right\rangle,
$$

where $\omega=e^{2 \pi i / p}$. This restricts to a free action on $S^{3}$ if $p$ and $q$ are relatively prime. The quotient of $S^{3}$ by this action is the lens space $L(p, q)$. In other words, there is a $p: 1$ covering $S^{3} \rightarrow L(p, q)$. Notice that $L(2,1)$ is the familiar projective space $\mathbb{R P}^{3}$.

For the Brieskorn sphere $\Sigma(p, q, r)$ with $p, q, r$ pairwise relatively prime, begin with the complex surface $Z$ defined by the equation

$$
z_{1}^{p}+z_{2}^{q}+z_{3}^{r}=0
$$

in $\mathbb{C}^{3}$. Let $S^{5}$ denote the unit 5 -sphere in $\mathbb{C}^{3}$. Then

$$
\Sigma(p, q, r)=Z \cup S^{5}
$$

Table 1: Asymptotic values of the Witten invariant for $L(12,5)$

Our first data in Table 1 is for the lens space $L(12,5)$. For some fairly large values of $k$ we display both the exact value of the path integral and the asymptotic value, as predicted by (53). (In fact, 
(53) must be modified to account for the fact that flat $S U_{2}$ connections on $L(12,5)$ are reducible.) This perturbative formula works out to be

$$
\begin{aligned}
Z_{L(12,5)}(k) \sim \frac{i}{2} \frac{\sqrt{2}}{\sqrt{12 \cdot(k+2)}} & \left\{4 \sin ^{2}\left(\frac{2}{12} \pi\right) e^{2 \pi i\left(\frac{5}{12}\right)(k+2)}\right. \\
& -4 \sin ^{2}\left(\frac{4}{12} \pi\right) e^{2 \pi i\left(\frac{8}{12}\right)(k+2)} \\
+ & 4 \sin ^{2}\left(\frac{6}{12} \pi\right) e^{2 \pi i\left(\frac{9}{12}\right)(k+2)} \\
& -4 \sin ^{2}\left(\frac{4}{12} \pi\right) e^{2 \pi i\left(\frac{8}{12}\right)(k+2)} \\
+ & \left.4 \sin ^{2}\left(\frac{2}{12} \pi\right) e^{2 \pi i\left(\frac{5}{12}\right)(k+2)}\right\} .
\end{aligned}
$$

Notice the different signs, which come from the spectral flow term. The numerical agreement observed in Table 1 has now been verified analytically [J].

Table 2: Asymptotic values of the Witten invariant for $\Sigma(2,3,17)$

Our second example is the Brieskorn sphere $\Sigma(2,3,17)$. This example is better since, except for the trivial connection, all flat $S U_{2}$ connections are irreducible and isolated points in the space of flat connections (up to equivalence). In fact, there are exactly 6 of them! So the asymptotic formula (53) 
works out to be

$$
\begin{aligned}
Z_{\Sigma(2,3,17)}(k) \sim \sqrt{\frac{2}{17}} e^{-3 \pi i / 4}\{ & \sin \left(\frac{\pi}{17}\right) e^{-\pi i(k+2) / 204} \\
& +\sin \left(\frac{4 \pi}{17}\right) e^{-169 \pi i(k+2) / 204} \\
& -\sin \left(\frac{8 \pi}{17}\right) e^{-217 \pi i(k+2) / 204} \\
& +\sin \left(\frac{3 \pi}{17}\right) e^{-145 \pi i(k+2) / 204} \\
& +\sin \left(\frac{2 \pi}{17}\right) e^{-361 \pi i(k+2) / 204} \\
& \left.-\sin \left(\frac{7 \pi}{17}\right) e^{-49 \pi i(k+2) / 204}\right\} .
\end{aligned}
$$

As you can see from Table 2, the exact solution is indeed asymptotic to (54), at least numerically. 


\section{REFERENCES}

[A] M. F. Atiyah, Topological quantum field theory, Publ. Math. Inst. Hautes Etudes Sci. (Paris) 68 (1989), $175-186$.

[ABR]. F. Atiyah, R. Bott, V. K. Patodi, On the heat equation and the index theorem, Invent. math. 19 (1973), $279-330$

[AD\$]Axelrod, S. Della Pietra, E. Witten, Geometric quantization of Chern-Simons gauge theory, J. Diff. Geo. 33 (1991), 787-902.

[APS]I. F. Atiyah, V. K. Patodi, I. M. Singer, Spectral asymmetry and Riemannian geometry. III, Math. Proc. Cambridge Philos. Soc. 79 (1976), 71-99.

[C] J. Cheeger, Analytic torsion and the heat equation, Ann. Math. 109 (1979), 259-322.

[CS]S. S. Chern, J. Simons, Characteristic forms and geometric invariants, Ann. Math. 99 (1974), 48-69.

[DWR. Dijkgraaf, E. Witten, Topological gauge theories and group cohomology, Commun. Math. Phys. 129 (1990), 393-429.

[DPR] Dijkgraaf, V. Pasquier, P. Roche, Quasi-quantum groups related to orbifold models, Nuclear Phys. B. Proc. Suppl. 18B (1991), 60-72.

[F1]D. S. Freed, Classical Chern-Simons theory, Part 1, Adv. Math. (to appear).

[F2]D. S. Freed, Higher line bundles (in preparation).

[F3]D. S. Freed, Classical Chern-Simons theory, Part 2 (in preparation).

[F4]D. S. Freed, Locality and integration in topological field theory, Proceedings of the XIX International Colloquium on Group Theoretical Methods in Physics, Ciemat (to appear).

[F5]D. S. Freed, Higher algebraic structures and quantization (preprint, 1992).

[FGD. S. Freed, R. E. Gompf, Computer calculation of Witten's 3-manifold invariant, Commun. Math. Phys. 141 (1991), 79-117.

[FGD]. S. Freed, R. E. Gompf, Computer tests of Witten's Chern-Simons theory against the theory of three-manifolds, Phys. Rev. Lett. 66 (1991), 1255-1258.

[FQD. S. Freed, F. Quinn, Chern-Simons theory with finite gauge group, Commun. Math. Phys. (to appear).

[Fr] W. Franz, Über die Torsion einer Überdeckung, J. Reine Angew. Math. 173 (1935), 245-254.

[G] S. Garoufalidis, Relations among 3-manifold invariants (University of Chicago Ph.D. thesis, 1992).

[GJ]J. Glimm, A. Jaffe, Quantum Physics. A functional integral point of view., Second edition, Springer-Verlag, New York-Berlin, 1987.

[GMR. Gompf, R. Mrowka, Irreducible four-manifolds need not be complex (preprint, 1991).

$[\mathrm{H}]$ N. Hitchin, Flat connections and geometric quantization, Commun. Math. Phys. 131 (1990), 347-380.

[J] L. C. Jeffery, On some aspects of Chern-Simons gauge theory (Oxford Univ. D.Phil. thesis, 1991).

[K] L. H. Kauffman, Knots and Physics, World Scientific, 1991.

[KMY. G. Kac, M. Wakimoto, Adv. Math. 70 (1988), 156.

[M] W. Müller, Analytic torsion and R-torsion of Riemannian manifolds, Adv. Math. 28 (1978), 233-305.

[Q1]F. Quinn, Lectures on axiomatic topological quantum field theory (preprint, 1992).

[Q2]F. Quinn, Topological foundations of topological quantum field theory (preprint, 1991).

[R] K. Reidemeister, Homotopieringe und Linsenräume, Hamburger Abhandl 11 (1935), 102-109.

[RS]D. B. Ray, I. M. Singer, R-torsion and the laplacian on Riemannian manifolds, Adv. Math. 7 (1971), 145-210.

[RSW] R. Ramadas, I. M. Singer, J. Weitsman, Some comments on Chern-Simons gauge theory, Commun. Math. Phys. 126 (1989), 409.

[RT]N. Y. Reshetikhin, V. G. Turaev, Ribbon graphs and their invariants derived from quantum groups, Commun. Math. Phys. 127 (1990), 1-26.

[S] G. Segal, The definition of conformal field theory (preprint).

[Sp] E. H. Spanier, Algebraic Topology, Springer-Verlag, New York, 1981.

[W1E. Witten, Quantum field theory and the Jones polynomial, Commun. Math. Phys. 121 (1989), 351-399.

[W2E. Witten, Topological sigma models, Commun. Math. Phys. 118 (1988), 411.

[Wa]K. Walker, On Witten's 3-manifold invariants (preprint, 1991).

Department of Mathematics, University of Texas, Austin, TX 78712

E-mail address: dafr@math.utexas.edu 